\title{
Lévy Measure Density Corresponding to Inverse Local Time
}

\author{
by
}

Tomoko TAKemura and Matsuyo Tomisaki

\begin{abstract}
We are concerned with the Lévy measure density corresponding to the inverse local time at the regular end point for a harmonic transform of a one-dimensional diffusion process. We show that the Lévy measure density is represented as the Laplace transform of the spectral measure corresponding to the original diffusion process, where the absorbing boundary condition is posed at the end point whenever it is regular.
\end{abstract}

2010 Mathematics Subject Classification: Primary 60G51; Secondary 60J55.

Keywords: inverse local time, Lévy measure, harmonic transformation.

\section{$\S 1$. Introduction}

Let $s$ be a continuous increasing function on an open interval $I=\left(l_{1}, l_{2}\right)$, where $-\infty \leq l_{1}<l_{2} \leq \infty$, let $m$ be a right continuous increasing function on $I$, and let $k$ be a right continuous nondecreasing function on $I$. Let $\mathcal{G}_{s, m, k}$ be a onedimensional diffusion operator on $I$ with scale function $s$, speed measure $m$, and killing measure $k$. We denote by $\mathbb{D}_{s, m, k}=\left[X(t), P_{x}\right]$ the one-dimensional diffusion process on $I$ with generator $\mathcal{G}_{s, m, k}$ and with end point $l_{i}$ where the absorbing boundary condition is posed whenever $l_{i}$ is $(s, m, k)$-regular $(i=1,2)$. For $\beta \geq 0$, let $\mathcal{H}_{s, m, k, \beta}$ be the set of all positive functions $h$ satisfying $\mathcal{G}_{s, m, k} h=\beta h$. For $h \in \mathcal{H}_{s, m, k, \beta}$, we set

$$
s_{h}(x)=\int_{\left(c_{o}, x\right]} h(y)^{-2} d s(y)
$$

Communicated by T. Kumagai. Received July 21, 2012. Revised February 4, 2013.

T. Takemura: Department of Mathematics, Nara Women's University, Kita-Uoya Nishimachi, Nara, 630-8506 Japan;

e-mail: sm18031@cc.nara-wu.ac.jp

M. Tomisaki: Department of Mathematics, Nara Women's University, Kita-Uoya Nishimachi, Nara, 630-8506 Japan;

e-mail: tomisaki@cc.nara-wu.ac.jp

(C) 2013 Research Institute for Mathematical Sciences, Kyoto University. All rights reserved. 


$$
m_{h}(x)=\int_{\left(c_{o}, x\right]} h(y)^{2} d m(y),
$$

where $c_{o} \in I$ is fixed arbitrarily. The diffusion operator $\mathcal{G}_{s_{h}, m_{h}, 0}$ with scale function $s_{h}$, speed measure $m_{h}$, and the null killing measure is the harmonic transform of $\mathcal{G}_{s, m, k}$ based on $h \in \mathcal{H}_{s, m, k, \beta}$. Let $\mathbb{D}_{s_{h}, m_{h}, 0}$ be the one-dimensional diffusion process on $I$ with generator $\mathcal{G}_{s_{h}, m_{h}, 0}$ and with the end point $l_{i}$ being absorbing whenever it is $\left(s_{h}, m_{h}, 0\right)$-regular $(i=1,2)$.

When $l_{1}$ is $\left(s_{h}, m_{h}, 0\right)$-regular, it is possible to pose the reflecting boundary condition at $l_{1}$. We denote by $\mathbb{D}_{s_{h}, m_{h}, 0}^{*}=\left[X(t), P_{x}^{(h *)}\right]$ such a diffusion process on $I$. Namely, the scale function and the speed measure are given by $s_{h}$ and $m_{h}$, respectively, the killing measure is null, and $l_{1}$ is $\left(s_{h}, m_{h}, 0\right)$-regular and reflecting. We consider the local time $l^{(h *)}(t, \xi)$ for $\mathbb{D}_{s_{h}, m_{h}, 0}^{*}$, that is,

$$
\int_{0}^{t} f(X(u)) d u=\int_{I} l^{(h *)}(t, \xi) d m_{h}(\xi), \quad t>0,
$$

for bounded continuous functions $f$ on $I$. Since $l^{(h *)}(t, \xi)$ is continuous and nondecreasing in $t P_{x}^{(h *)}$-a.s., the right continuous inverse function $l^{(h *)^{-1}}(t, \xi)$ exists. In particular, we denote by $\tau^{(h *)}(t)$ the inverse local time $l^{(h *)^{-1}}\left(t, l_{1}\right)$ at the end point $l_{1}$. Employing some results due to Itô and McKean (see [5, Section 6.2]), we find that, if $s_{h}\left(l_{2}\right)=\infty$, then $\left[\tau^{(h *)}(t), t \geq 0\right]$ is a Lévy process and there is a Lévy measure density $n^{(h *)}(\xi)$ such that

$$
E_{l_{1}}^{(h *)}\left[e^{-\lambda \tau^{(h *)}(t)}\right]=\exp \left\{-t \int_{0}^{\infty}\left(1-e^{-\lambda \xi}\right) n^{(h *)}(\xi) d \xi\right\},
$$

where $E_{l_{1}}^{(h *)}$ stands for the expectation with respect to $P_{l_{1}}^{(h *)}$. The aim of this paper is to give a representation of $n^{(h *)}(\xi)$ in terms of data corresponding to the diffusion process $\mathbb{D}_{s, m, k}$. We state our results in Section 3 (see Theorem 3.2).

Applying our results, we find some interesting facts. Let us consider the following diffusion generators on $(0, \infty)$ :

$$
\begin{aligned}
& \mathcal{L}_{1}=\frac{1}{2} \frac{d^{2}}{d x^{2}}+\left\{\frac{1}{2 x}+\sqrt{2 \beta} \frac{K_{\nu}^{\prime}(\sqrt{2 \beta} x)}{K_{\nu}(\sqrt{2 \beta} x)}\right\} \frac{d}{d x}, \\
& \mathcal{L}_{2}=\frac{1}{2} \frac{d^{2}}{d x^{2}}+\left\{-\frac{1}{2 x}+2 \kappa x \frac{W_{-\frac{\beta}{2 \kappa}+\frac{\nu+1}{2}, \frac{|\nu|}{2}}^{\prime}\left(\kappa x^{2}\right)}{W_{-\frac{\beta}{2 \kappa}+\frac{\nu+1}{2}, \frac{|\nu|}{2}}\left(\kappa x^{2}\right)}\right\} \frac{d}{d x}, \\
& \mathcal{L}_{3}=\frac{1}{2} \frac{d^{2}}{d x^{2}}+\left\{-\frac{1}{2 x}+2 \kappa x \frac{W_{-\frac{\beta}{2 \kappa}-\frac{\nu+1}{2}, \frac{|\nu|}{2}}^{\prime}\left(\kappa x^{2}\right)}{W_{-\frac{\beta}{2 \kappa}-\frac{\nu+1}{2}, \frac{|\nu|}{2}}\left(\kappa x^{2}\right)}\right\} \frac{d}{d x},
\end{aligned}
$$

where $-1<\nu<1, \kappa>0$ and $\beta>0 . K_{l}(x)$ and $W_{k, l}(x)$ are the modified Bessel function and the Whittaker function, respectively (see Section 4 for their 
definitions). Let $\mathbb{D}_{i}^{*}$ be the diffusion process on $(0, \infty)$ with generator $\mathcal{L}_{i}(i=$ $1,2,3)$. The end point 0 is regular for all $\mathbb{D}_{i}^{*}$. Therefore the inverse local time $\tau^{*}(t)$ at the end point 0 with reflecting boundary condition exists. Noting that $\mathcal{L}_{1}$ is a harmonic transform of a Bessel operator, and $\mathcal{L}_{2}$ and $\mathcal{L}_{3}$ are harmonic transforms of radial Ornstein-Uhlenbeck operators, by means of Theorem 3.2 below, we find that the Lévy measure densities $n_{i}^{*}(\xi)$ of $\tau^{*}(t)$ corresponding to $\mathbb{D}_{i}^{*}$ are

$$
\begin{aligned}
& n_{1}^{*}(\xi)=C \xi^{-(|\nu|+1)} e^{-\beta \xi}, \\
& n_{2}^{*}(\xi)=C\left(\frac{\kappa}{\sinh (\kappa \xi)}\right)^{|\nu|+1} e^{\{\kappa(\nu+1)-\beta\} \xi}, \\
& n_{3}^{*}(\xi)=C\left(\frac{\kappa}{\sinh (\kappa \xi)}\right)^{|\nu|+1} e^{\{-\kappa(\nu+1)-\beta\} \xi},
\end{aligned}
$$

where $C=2^{-(|\nu|+1)} \Gamma(|\nu|+1)$. In Section 2, we show a simple convergence theorem for a sequence of Lévy measure densities under the assumption that the scale functions and speed measures are convergent (see Theorem 2.4). Since the scale functions and speed measures of $\mathcal{L}_{2}$ and $\mathcal{L}_{3}$ converge to the scale function and speed measure of $\mathcal{L}_{1}$ as $\kappa \rightarrow 0$, respectively, Theorem 2.4 leads to

$$
n_{1}^{*}(\xi)=\lim _{\kappa \rightarrow 0} n_{2}^{*}(\xi)=\lim _{\kappa \rightarrow 0} n_{3}^{*}(\xi) .
$$

This also directly follows from (1.6)-(1.8).

We next consider the following diffusion operator on $(0, a)$ :

$$
\mathcal{L}_{4}=\frac{1}{2} \frac{d^{2}}{d x^{2}}-\sqrt{2 \beta} \frac{e^{2 \sqrt{2 \beta} a}+e^{2 \sqrt{2 \beta} x}}{e^{2 \sqrt{2 \beta} a}-e^{2 \sqrt{2 \beta} x}} \frac{d}{d x}
$$

where $a>0$ and $\beta>0$. Let $\mathbb{D}_{4}^{*}$ be the diffusion process on $(0, a)$ with generator $\mathcal{L}_{4}$. The end point 0 is regular and the inverse local time $\tau^{*}(t)$ at the end point 0 with reflecting boundary condition exists. Since $\mathcal{L}_{4}$ is a harmonic transform of Brownian motion on $(0, a)$, by using Theorem 3.2 we find that the Lévy measure density of $\tau^{*}(t)$ is

$$
n_{4}^{*}(\xi)=a^{-3} e^{-\beta \xi} \sum_{n=1}^{\infty}(n \pi)^{2} e^{-\left(n^{2} \pi^{2} / 2 a^{2}\right) \xi} .
$$

It is easy to see that $\mathcal{L}_{4}$ converges to $\tilde{\mathcal{L}}_{4}=\frac{1}{2} \frac{d^{2}}{d x^{2}}-\sqrt{2 \beta} \frac{d}{d x}$ as $a \rightarrow \infty$, in the sense that the scale function and the speed measure of $\mathcal{L}_{4}$ converge to those of $\tilde{\mathcal{L}}_{4}$. Therefore by Theorem 2.4, we find that

$$
\lim _{a \rightarrow \infty} n_{4}^{*}(\xi)=\tilde{n}_{4}^{*}(\xi)=\sqrt{\pi / 2} e^{-\beta \xi} \xi^{-3 / 2},
$$


where $\tilde{n}_{4}^{*}$ is the Lévy measure density of the inverse local time corresponding to $\tilde{\mathcal{L}}_{4}$. (1.11) also follows from (1.10).

In [2] and [3], C. Donati-Martin and M. Yor considered the diffusion processes $\mathbb{D}_{i}^{*}(i=1,2)$, and showed (1.6), and (1.7) with $-1<\nu<0$. Their method is based on various transforms of Bessel processes. In [12] the second author gave an analytical proof for the representation (1.7) by using a harmonic transform. In this paper we generalize the analytical method used in [12] to obtain Theorem 3.2.

In Section 2 we summarize some definitions and facts needed below. Then we prove a convergence theorem for a sequence of Lévy measure densities. In Section 3 we state our main results and prove them. In Section 4 we present some interesting diffusion operators including the $\mathcal{L}_{i}$ 's given by (1.3)-(1.5) and (1.9).

\section{§2. Preliminaries}

Let $s, m$, and $k$ be the functions given at the beginning of the previous section. We sometimes use the same symbols $s, m$ and $k$ for the respective induced measures $d s(x), d m(x)$ and $d k(x)$. For a function $u$ on $I$, we set $u\left(l_{i}\right)=\lim _{x \rightarrow l_{i}, x \in I} u(x)$ if the limit exists, for $i=1,2$. We denote by $D_{s} u(x)$ the right derivative with respect to $s(x)$, that is, $D_{s} u(x)=\lim _{\varepsilon \downarrow 0}\{u(x+\varepsilon)-u(x)\} /\{s(x+\varepsilon)-s(x)\}$, provided it exists. Let us fix a point $c_{o} \in I$ arbitrarily and set

$$
J_{\mu, \nu}(x)=\int_{\left(c_{o}, x\right]} d \mu(y) \int_{\left(c_{o}, y\right]} d \nu(z) \quad \text { for } x \in I,
$$

where $\mu$ and $\nu$ are Borel measures on $I$, and the integral $\int_{(a, b]}$ is read as $-\int_{(b, a]}$ if $a>b$. Following [4], we call the boundary point $l_{i}$

- $(s, m, k)$-regular if $J_{s, m+k}\left(l_{i}\right)<\infty$ and $J_{m+k, s}\left(l_{i}\right)<\infty$,

- an $(s, m, k)$-exit point if $J_{s, m+k}\left(l_{i}\right)<\infty$ and $J_{m+k, s}\left(l_{i}\right)=\infty$,

- an $(s, m, k)$-entrance point if $J_{s, m+k}\left(l_{i}\right)=\infty$ and $J_{m+k, s}\left(l_{i}\right)<\infty$,

- $(s, m, k)$-natural if $J_{s, m+k}\left(l_{i}\right)=\infty$ and $J_{m+k, s}\left(l_{i}\right)=\infty$.

\section{§2.1. One-dimensional diffusion process $\mathbb{D}_{s, m, k}$}

Let $D\left(\mathcal{G}_{s, m, k}\right)$ be the space of all functions $u \in L^{2}(I, m)$ which have a continuous version $u$ (we use the same symbol) satisfying the following conditions:

$\left(\mathcal{G}\right.$-i) There exist constants $A, B$ and a function $f_{u} \in L^{2}(I, m)$ such that

$$
\begin{aligned}
u(x)= & A+B s(x)+\int_{\left(c_{o}, x\right]}\{s(x)-s(y)\} f_{u}(y) d m(y) \\
& +\int_{\left(c_{o}, x\right]}\{s(x)-s(y)\} u(y) d k(y), \quad x \in I .
\end{aligned}
$$


$\left(\mathcal{G}\right.$-ii) If $l_{i}$ is $(s, m, k)$-regular, then $u\left(l_{i}\right)=0$ for each $i=1,2$.

By (2.1), $f_{u}$ is uniquely determined as a function in $L^{2}(I, m)$ if it exists. The operator $\mathcal{G}_{s, m, k}$ from $D\left(\mathcal{G}_{s, m, k}\right)$ into $L^{2}(I, m)$ is defined by $\mathcal{G}_{s, m, k} u=f_{u}$, and it is called the one-dimensional generalized diffusion operator with scale function $s$, speed measure $m$, and killing measure $k$. The condition $(\mathcal{G}$-ii) implies that the absorbing boundary condition is posed at the regular boundary.

We denote by $\mathbb{D}_{s, m, k}=\left[X(t), P_{x}\right]$ the one-dimensional diffusion process on $I$ whose generator is $\mathcal{G}_{s, m, k}$ with domain $D\left(\mathcal{G}_{s, m, k}\right)$, that is, the end point $l_{i}$ is absorbing whenever it is $(s, m, k)$-regular $(i=1,2)$. Further we denote by $p(t, x, y)$ the transition probability density with respect to $d m$ for $\mathbb{D}_{s, m, k}$, that is,

$$
P_{x}(X(t) \in E)=\int_{E} p(t, x, y) d m(y), \quad t>0, x \in I, E \in \mathcal{B}(I),
$$

where $\mathcal{B}(I)$ stands for the set of all Borel subsets of $I$. If $l_{1}$ is $(s, m, k)$-regular, $p(t, x, y)$ is represented as

$$
p(t, x, y)=\int_{[0, \infty)} e^{-\lambda t} \psi_{o}(x, \lambda) \psi_{o}(y, \lambda) d \sigma(\lambda), \quad t>0, x, y \in I,
$$

where $d \sigma(\lambda)$ is a Borel measure on $[0, \infty)$ satisfying

$$
\int_{[0, \infty)} e^{-\lambda t} d \sigma(\lambda)<\infty, \quad t>0
$$

and $\psi_{o}(x, \lambda), x \in I, \lambda \geq 0$, is the solution of the integral equation

$$
\psi_{o}(x, \lambda)=s(x)-s\left(l_{1}\right)+\int_{\left(l_{1}, x\right]}\{s(x)-s(y)\} \psi_{o}(y, \lambda)\{-\lambda d m(y)+d k(y)\} .
$$

It is well known that (2.4) has a unique solution. If $l_{1}$ is not $(s, m, k)$-regular, $p(t, x, y)$ is not always representable as in (2.2) with $d \sigma(\lambda)$ satisfying (2.3) and $\psi_{o}(x, \lambda)$ the solution of an integral equation. If $l_{1}$ is an $(s, m, k)$-entrance point, we give a sufficient condition for $p(t, x, y)$ to have a representation $(2.2)$. Let $\psi(x, \lambda)$, $x \in I, \lambda \geq 0$, be the solution of the integral equation

$$
\psi(x, \lambda)=1+\int_{\left(l_{1}, x\right]}\{s(x)-s(y)\} \psi(y, \lambda)\{-\lambda d m(y)+d k(y)\} .
$$

Proposition 2.1. Assume that $l_{1}$ is an $(s, m, k)$-entrance point and

$$
\int_{\left(l_{1}, c_{o}\right]}\left\{s\left(c_{o}\right)-s(x)\right\}^{2} d m(x)<\infty .
$$

Then $p(t, x, y)$ can be represented as in (2.2) with $d \sigma(\lambda)$ satisfying (2.3) and $\psi_{o}(x, \lambda)$ replaced by $\psi(x, \lambda)$ which is the solution of the integral equation (2.5). 
We show Proposition 2.1 in Section 2.4.

We next record some estimates for the solution of (2.4) or (2.5). If $l_{1}$ is $(s, m, k)$-regular or an $(s, m, k)$-exit point, and $\psi_{o}(x, \lambda)$ is the solution of $(2.4)$, then

$$
\begin{aligned}
\left|\psi_{o}(x, \lambda)\right| & \leq\left\{s(x)-s\left(l_{1}\right)\right\} \exp \left\{\int_{\left(l_{1}, x\right]} d s(y) \int_{(y, x]}(\lambda d m(z)+d k(z))\right\}, \\
\left|D_{s} \psi_{o}(x, \lambda)\right| & \leq \exp \left\{\int_{\left(l_{1}, x\right]} d s(y) \int_{(y, x]}(\lambda d m(z)+d k(z))\right\} .
\end{aligned}
$$

If $l_{1}$ is an $(s, m, k)$-entrance point, and $\psi(x, \lambda)$ is the solution of $(2.5)$, then

$$
|\psi(x, \lambda)| \leq \exp \left\{\int_{\left(l_{1}, x\right]}(\lambda d m(y)+d k(y)) \int_{(y, x]} d s(z)\right\}
$$

$$
\left|D_{s} \psi(x, \lambda)\right| \leq \int_{\left(l_{1}, x\right]}(\lambda d m(y)+d k(y)) \exp \left\{\int_{\left(l_{1}, x\right]}(\lambda d m(y)+d k(y)) \int_{(y, x]} d s(z)\right\} .
$$

It is easy to get (2.7) with (2.10), so we omit the proof.

For $\alpha \geq 0$ and $i=1,2$, let $g_{i}(\cdot, \alpha)$ be a function on $I$ with the following properties:

(2.11) $g_{i}(x, \alpha)$ is positive and continuous in $x$,

(2.12) $g_{1}(x, \alpha)$ is nondecreasing in $x$,

(2.13) $g_{2}(x, \alpha)$ is nonincreasing in $x$,

(2.14) $\quad g_{i}\left(l_{i}, \alpha\right)=0$ if $\left|s\left(l_{i}\right)\right|<\infty$,

(2.15) $\quad g_{i}(x, \alpha)=g_{i}\left(c_{o}, \alpha\right)+D_{s} g_{i}\left(c_{o}, \alpha\right)\left\{s(x)-s\left(c_{o}\right)\right\}$

$$
+\int_{\left(c_{o}, x\right]}\{s(x)-s(y)\} g_{i}(y, \alpha)\{\alpha d m(y)+d k(y)\}, \quad x \in I .
$$

Here $D_{s} g_{i}(x, \alpha)=\lim _{\varepsilon \downarrow 0}\left\{g_{i}(x+\varepsilon, \alpha)-g_{i}(x, \alpha)\right\} /\{s(x+\varepsilon)-s(x)\}, i=1,2$. It is known that there exist functions with the above properties (see [5, Section 4.6]).

Combining some properties of $g_{i}(x, \alpha)$ given in [5, Section 4.6] with Lemma 3.2 of [11], we obtain the following.

Proposition 2.2. Assume that $k$ is not a null measure or $\alpha>0$. Then

$$
\begin{aligned}
& \left|\int_{\left(l_{i}, c_{o}\right]} g_{i}(x, \alpha)^{-2} d s(x)\right|=\infty, \\
& \left|\int_{\left(l_{i}, c_{o}\right]} g_{j}(x, \alpha)^{-2} d s(x)\right|<\infty,
\end{aligned}
$$

where $i, j=1,2$ and $i \neq j$. 
Now we set $W(\alpha)=D_{s} g_{1}(x, \alpha) g_{2}(x, \alpha)-g_{1}(x, \alpha) D_{s} g_{2}(x, \alpha)$. Note that $W(\alpha)$ is a positive number independent of $x \in I$. We put

$$
G(\alpha, x, y)=G(\alpha, y, x)=W(\alpha)^{-1} g_{1}(x, \alpha) g_{2}(y, \alpha)
$$

for $\alpha>0, x, y \in I, x \leq y$. Then $G(\alpha, x, y)$ is the $\alpha$-Green function corresponding to $\mathbb{D}_{s, m, k}$ and

$$
G(\alpha, x, y)=\int_{0}^{\infty} e^{-\alpha t} p(t, x, y) d t, \quad \alpha>0, x, y \in I .
$$

It is easy to see that, if $k \neq 0$, then $G(0, x, y)$ exists and is given by

$$
G(0, x, y)=G(0, y, x)=W^{-1} g_{1}(x) g_{2}(y), \quad x, y \in I, x \leq y,
$$

where $g_{i}(x)=g_{i}(x, 0), i=1,2$, and $W=D_{s} g_{1}(x) g_{2}(x)-g_{1}(x) D_{s} g_{2}(x)$, which is a positive constant independent of $x \in I$. In the case $k=0, G(0, x, y) \in(0, \infty)$ exists if and only if $\left|s\left(l_{i}\right)\right|<\infty$ for $i=1$ or 2 .

\section{$\S 2.2$. Inverse local time}

We next consider the case that the end point $l_{i}$ is reflecting whenever it is $(s, m, k)$ regular $(i=1,2)$. More precisely, let $D\left(\mathcal{G}_{s, m, k}^{*}\right)$ be the space of all functions $u \in L^{2}(I, m)$ which have a continuous version $u$ (we use the same symbol) satisfying $(\mathcal{G}$-i) and

$\left(\mathcal{G}\right.$-iii) If $l_{i}$ is $(s, m, k)$-regular, then $D_{s} u\left(l_{i}\right)=0$ for each $i=1,2$.

(2.1) implies that $f_{u}$ is uniquely determined as a function in $L^{2}(I, m)$ if it exists, in this case, too. The operator $\mathcal{G}_{s, m, k}^{*}$ from $D\left(\mathcal{G}_{s, m, k}^{*}\right)$ into $L^{2}(I, m)$ is defined by $\mathcal{G}_{s, m, k}^{*} u=f_{u}$. The condition $(\mathcal{G}$-iii) implies that the reflecting boundary condition is posed at the regular boundary.

Let $\mathbb{D}_{s, m, k}^{*}=\left[X(t), P_{x}^{*}\right]$ be the one-dimensional diffusion process on $I$ whose generator is $\mathcal{G}_{s, m, k}^{*}$ with domain $D\left(\mathcal{G}_{s, m, k}^{*}\right)$, that is, the end point $l_{i}$ is reflecting whenever it is $(s, m, k)$-regular $(i=1,2)$.

Now we assume that the killing measure is null, and $l_{1}$ is $(s, m, 0)$-regular. We consider the local time $l(t, \xi)$, that is,

$$
\int_{0}^{t} f(X(u)) d u=\int_{I} l(t, \xi) d m(\xi), \quad t>0,
$$

for bounded continuous functions $f$ on $I$. Since $l(t, \xi)$ is continuous and nondecreasing in $t P_{x}^{*}$-a.s., the right continuous inverse function $l^{-1}(t, \xi)$ exists. In particular, we denote by $\tau^{*}(t)$ the inverse local time $l^{-1}\left(t, l_{1}\right)$ at the end point $l_{1}$. The following result was obtained by Itô and McKean (see [5, Section 6.2]). 
Proposition 2.3 ([5]). Assume the following conditions hold: The killing measure is null.

$$
l_{1} \text { is }(s, m, 0) \text {-regular and reflecting. }
$$

Then $\left[\tau^{*}(t), t \geq 0\right]$ is a Lévy process and there is a Lévy measure density $n^{*}(\xi)$ such that

$$
\begin{aligned}
E_{l_{1}}^{*}\left[e^{-\lambda \tau^{*}(t)}\right] & =\exp \left\{-t \int_{0}^{\infty}\left(1-e^{-\lambda \xi}\right) n^{*}(\xi) d \xi\right\}, \\
n^{*}(\xi) & =\lim _{x \rightarrow l_{1}} q^{*}(\xi, x) /\left\{s(x)-s\left(l_{1}\right)\right\}
\end{aligned}
$$

where $E_{l_{1}}^{*}$ stands for the expectation with respect to $P_{l_{1}}^{*}$,

$$
\int_{0}^{t} q^{*}(\xi, x) d \xi=P_{x}^{*}\left(\sigma_{l_{1}}<t\right), \quad x \in I, t>0
$$

and $\sigma_{l_{1}}$ is the first hitting time for $l_{1}$. In particular, if $s\left(l_{2}\right)=\infty$, then

$$
n^{*}(\xi)=\lim _{x, y \rightarrow l_{1}} D_{s(x)} D_{s(y)} p(\xi, x, y)=\int_{[0, \infty)} e^{-\lambda \xi} d \sigma(\lambda)
$$

where $p(t, x, y)$ is the transition probability density with respect to $d m$ for $\mathbb{D}_{s, m, k}$, and $d \sigma(\lambda)$ is the Borel measure appearing in the representation (2.2) satisfying $(2.3)$.

Proof. In Section 6.2 of [5], (2.21) with (2.22) is obtained under the assumption

$$
P_{x}^{*}\left(\sigma_{l_{1}}<\infty\right)=1, \quad x \in I
$$

In view of Problem 4.6.6 of [5], (2.25) holds true if and only if (2.18) and (2.20) are satisfied. Thus we obtain (2.21) and (2.22).

Assume $s\left(l_{2}\right)=\infty$. Then

$$
q^{*}(t, x)=\lim _{z \rightarrow l_{1}} p(t, z, x) /\left\{s(z)-s\left(l_{1}\right)\right\}, \quad t>0, x \in I .
$$

Since $p(t, x, y)$ has the representation $(2.2)$ and $\psi_{o}(x, \lambda)$ is the solution of $(2.4)$, we have

$$
\begin{aligned}
& \lim _{x \rightarrow l_{1}} D_{s} \psi_{o}(x, \lambda)=1 \\
& \left|D_{s} \psi_{o}(x, \lambda)\right| \leq \exp \left\{\lambda\left(s(x)-s\left(l_{1}\right)\right)\left(m(x)-m\left(l_{1}\right)\right)\right\}
\end{aligned}
$$


by means of (2.8). Therefore we obtain (2.24) by virtue of $(2.22),(2.26)$, and the dominated convergence theorem.

We next give a simple convergence theorem for a sequence of Lévy measure densities. For $j=0,1,2, \ldots$, let $\mathbb{D}^{* j}$ be the diffusion process on $I^{j}=\left(l_{1}^{j}, l_{2}^{j}\right)$ with scale function $s^{j}$, speed measure $m^{j}$ and no killing.

Theorem 2.4. Assume the following conditions hold:

$$
\begin{aligned}
& \lim _{j \rightarrow \infty} l_{i}^{j}=l_{i}^{0}, \quad i=1,2, \\
& \lim _{j \rightarrow \infty} s^{j}(x)=s^{0}(x), \quad x \in I^{0}, \\
& \lim _{j \rightarrow \infty} m^{j}(x)=m^{0}(x), \quad x \in \mathcal{C}\left(m^{0}\right),
\end{aligned}
$$

where $\mathcal{C}\left(m^{0}\right)$ stands for the set of continuity points of $m^{0}$. Further for $j=0,1,2, \ldots$, assume that

$$
l_{1}^{j} \text { is }\left(s^{j}, m^{j}, 0\right) \text {-regular and reflecting, } \quad s^{j}\left(l_{2}^{j}\right)=\infty \text {. }
$$

Let $n^{* j}$ be the Lévy measure density corresponding to the inverse local time at $l_{1}^{j}$ for $\mathbb{D}^{* j}$. Then

$$
\lim _{j \rightarrow \infty} n^{* j}(\xi)=n^{* 0}(\xi), \quad \xi>0 .
$$

Proof. For $j=0,1,2, \ldots$, let $\mathbb{D}^{j}$ be the diffusion process on $I^{j}$ with scale function $s^{j}$, speed measure $m^{j}$ and no killing, and with $l_{1}^{j}$ being absorbing. The transition probability density $p^{j}(t, x, y)$ with respect to $d m^{j}$ is represented as

$$
p^{j}(t, x, y)=\int_{[0, \infty)} e^{-\lambda t} \psi_{o}^{j}(x, \lambda) \psi_{o}^{j}(y, \lambda) d \sigma^{j}(\lambda), \quad t>0, x, y \in I^{j}
$$

where $d \sigma^{j}(\lambda)$ satisfies (2.3) with $\sigma$ replaced by $\sigma^{j}$, and $\psi_{o}^{j}$ is the solution of (2.4) with $s=s^{j}, m=m^{j}$, and $k=0$. By Proposition 2.3, $n^{* j}$ is represented as (2.24), that is,

$$
n^{* j}(\xi)=\int_{[0, \infty)} e^{-\lambda \xi} d \sigma^{j}(\lambda)
$$

In the same way as in the proof of Lemma 5.3 of [8], we obtain

$$
\lim _{j \rightarrow \infty} \int_{[0, \infty)} e^{-\lambda \xi} d \sigma^{j}(\lambda)=\int_{[0, \infty)} e^{-\lambda \xi} d \sigma^{0}(\lambda)
$$

which implies (2.27). 


\section{§2.3. Harmonic transform of $\mathcal{G}_{s, m, k}$}

For $\beta \geq 0$, let $h_{\beta}(\cdot)$ be a positive continuous function on $I$ satisfying

$$
\begin{aligned}
h_{\beta}(x)= & h_{\beta}\left(c_{o}\right)+D_{s} h_{\beta}\left(c_{o}\right)\left\{s(x)-s\left(c_{o}\right)\right\} \\
& +\int_{\left(c_{o}, x\right]}\{s(x)-s(y)\} h_{\beta}(y)\{\beta d m(y)+d k(y)\}, \quad x \in I .
\end{aligned}
$$

Such a function exists. Indeed, it can be represented as a linear combination of $g_{i}(\cdot, \beta), i=1,2$.

Let $\mathcal{H}_{s, m, k, \beta}$ be the set of all positive functions $h_{\beta}$ satisfying (2.28). For $h \in \mathcal{H}_{s, m, k, \beta}$, we consider $s_{h}$ and $m_{h}$ defined by (1.1) and (1.2), respectively. Let $\mathcal{G}_{s_{h}, m_{h}, 0}$ be the diffusion operator with scale function $s_{h}$, speed measure $m_{h}$, and null killing measure, which is the harmonic transform of $\mathcal{G}_{s, m, k}$ based on $h \in \mathcal{H}_{s, m, k, \beta}$. Let $\mathbb{D}_{s_{h}, m_{h}, 0}$ be the one-dimensional diffusion process on $I$ with generator $\mathcal{G}_{s_{h}, m_{h}, 0}$ and with the end point $l_{i}$ being absorbing whenever it is $\left(s_{h}, m_{h}, 0\right)$ regular $(i=1,2)$. Further we denote by $p^{(h)}(t, x, y)$ the transition probability density with respect to $d m_{h}$ for $\mathbb{D}_{s_{h}, m_{h}, 0}$. By Proposition 2.2 of [11],

$$
p^{(h)}(t, x, y)=e^{-\beta t} p(t, x, y) / h(x) h(y), \quad t>0, x, y \in I .
$$

If $l_{1}$ is $\left(s_{h}, m_{h}, 0\right)$-regular, $p^{(h)}(t, x, y)$ is represented as

$$
p^{(h)}(t, x, y)=\int_{[0, \infty)} e^{-\lambda t} \psi_{o}^{(h)}(x, \lambda) \psi_{o}^{(h)}(y, \lambda) d \sigma^{(h)}(\lambda), \quad t>0, x, y \in I,
$$

where $d \sigma^{(h)}(\lambda)$ is a Borel measure on $[0, \infty)$ satisfying

$$
\int_{[0, \infty)} e^{-\lambda t} d \sigma^{(h)}(\lambda)<\infty, \quad t>0,
$$

and $\psi_{o}^{(h)}(x, \lambda), x \in I, \lambda \geq \beta$, is the solution of the integral equation (2.4) with $s, m, k$ replaced by $s_{h}, m_{h}, 0$, respectively.

Remark 2.5. We can show $d \sigma^{(h)}(\lambda)=0$ on $[0, \beta)$. The proof is the same as the proof of Proposition 2.1 below, so we omit it.

\section{§2.4. Proof of Proposition 2.1}

First we note the following.

Lemma 2.6. Assume that $l_{1}$ is an $(s, m, k)$-entrance point. Then the following conditions are equivalent:

(i) (2.6) holds true. 
(ii) There exists a $\beta>0$ such that

$$
\int_{\left(l_{1}, c_{o}\right]} g_{2}(x, \beta)^{2} d m(x)<\infty .
$$

(iii) For any $\beta>0$,

$$
\int_{\left(l_{1}, c_{o}\right]} g_{2}(x, \beta)^{2} d m(x)<\infty .
$$

(iv) There exist $\beta>0$ and $h \in \mathcal{H}_{s, m, k, \beta}$ such that $h\left(l_{1}\right)=\infty$ and $\left|m_{h}\left(l_{1}\right)\right|<\infty$.

(v) $\left|m_{h}\left(l_{1}\right)\right|<\infty$ for any $\beta>0$ and $h \in \mathcal{H}_{s, m, k, \beta}$.

Proof. Since $l_{1}$ is an $(s, m, k)$-entrance point, $\left|m_{h}\left(l_{1}\right)\right|<\infty$ and $\left|D_{s} g_{2}\left(l_{1}, \beta\right)\right| \in$ $(0, \infty)$ for $\beta>0$ (see [5, Section 4.6]). Therefore (i) $\Leftrightarrow($ ii) $\Leftrightarrow($ iii) follows.

Let $h \in \mathcal{H}_{s, m, k, \beta}$. Since $h$ can be represented as a linear combination of $g_{i}(\cdot, \beta), i=1,2$, and $g_{2}\left(l_{1}, \beta\right)=\infty$,

$$
h\left(l_{1}\right)=\infty \Leftrightarrow h(x)=C_{1} g_{1}(x, \beta)+C_{2} g_{2}(x, \beta) \text { with } C_{1} \geq 0 \text { and } C_{2}>0 .
$$

Therefore (ii) $\Leftrightarrow($ iv $) \Leftrightarrow(\mathrm{v})$ follows.

Remark 2.7. If $k \neq 0$ or $\beta>0$, then

$$
\int_{\left(l_{1}, c_{o}\right]} g_{1}(x, \beta)^{2} d m(x)<\infty, \quad g_{2}\left(l_{1}, \beta\right)=\infty, \quad\left|D_{s} g_{2}\left(l_{1}, \beta\right)\right| \in(0, \infty) .
$$

If $k=0$ and $\beta=0$, then

$$
g_{1}(x, 0)=C_{1}, \quad g_{2}(x, 0)= \begin{cases}C_{2} & \text { if } s\left(l_{2}\right)=\infty, \\ C_{2}\left\{s\left(l_{2}\right)-s(x)\right\} & \text { if } s\left(l_{2}\right)<\infty,\end{cases}
$$

where $C_{1}$ and $C_{2}$ are positive constants (see [5, Section 4.6]; see also [10, Proposition 2.1]). Therefore the lemma holds true with $\beta \geq 0$ in place of $\beta>0$ in (iii)-(v). In particular, if $k \neq 0$ or $s\left(l_{2}\right)<\infty$, the lemma holds true with $\beta \geq 0$ in place of $\beta>0$ in (ii) $-(\mathrm{v})$.

Proof of Proposition 2.1. Assume that $l_{1}$ is an $(s, m, k)$-entrance point, and (2.6) holds. By Lemma 2.6, there exist $\beta>0$ and $h \in \mathcal{H}_{s, m, k, \beta}$ such that $h\left(l_{1}\right)=\infty$ and $\left|m_{h}\left(l_{1}\right)\right|<\infty$. Then $l_{1}$ is $\left(s_{h}, m_{h}, 0\right)$-regular by Theorem 1.1 of [9]. Therefore $p^{(h)}(t, x, y)$ is represented as $(2.30)$ with $d \sigma^{(h)}(\lambda)$ satisfying $(2.31)$ and $\psi_{o}^{(h)}(x, \lambda)$, $x \in I, \lambda \geq 0$, the solution of the integral equation (2.4) with $s, m, k$ replaced by $s_{h}, m_{h}, 0$, respectively. First we note that

$$
\int_{[0, \beta)} d \sigma^{(h)}(\lambda)=0 .
$$


Indeed, if $\int_{[0, \beta)} d \sigma^{(h)}(\lambda)>0$, then by $(2.29)$,

$$
\begin{aligned}
p(t, x, x) & =e^{\beta t} h(x)^{2} p^{(h)}(t, x, x) \\
& \geq h(x)^{2} \int_{[0, \beta)} e^{(\beta-\lambda) t} \psi_{o}^{(h)}(x, \lambda)^{2} d \sigma^{(h)}(\lambda) \rightarrow \infty \quad \text { as } t \rightarrow \infty .
\end{aligned}
$$

This contradicts the existence of $\lim _{t \rightarrow \infty} p(t, x, x) \in[0, \infty)$.

By (2.30) and (2.32), we have

$$
p(t, x, y)=\int_{[0, \infty)} e^{-\lambda t} \widetilde{\psi}(x, \lambda) \widetilde{\psi}(y, \lambda) d \widetilde{\sigma}(\lambda),
$$

where $\widetilde{\psi}(x, \lambda)=h(x) \psi_{o}^{(h)}(x, \lambda+\beta)$ and $d \widetilde{\sigma}(\lambda)=d_{\lambda} \sigma^{(h)}(\lambda+\beta)$. By (2.4) and Lemma 5.1 of [10],

$$
\begin{aligned}
\widetilde{\psi}\left(l_{1}, \lambda\right) & =-1 / D_{s} h\left(l_{1}\right) \in(0, \infty), \\
D_{s} \widetilde{\psi}\left(l_{1}, \lambda\right) & =0 .
\end{aligned}
$$

Since $h(x)$ satisfies $(2.28)$ and $\psi_{o}^{(h)}(\xi, \lambda+\beta)$ satisfies (2.4) with $s, m, k, \lambda$ replaced by $s_{h}, m_{h}, 0, \lambda+\beta$, respectively, we have

$$
D_{s} \widetilde{\psi}(y, \lambda)-D_{s} \widetilde{\psi}(x, \lambda)=\int_{(x, y]} \widetilde{\psi}(\xi, \lambda)\{-\lambda d m(\xi)+d k(\xi)\}
$$

for $l_{1}<x \leq y<l_{2}$. Noting $\int_{[0, \infty)} e^{-\lambda t} d \widetilde{\sigma}(\lambda)<\infty$ and putting $\psi(x, \lambda)=$ $-D_{s} h\left(l_{1}\right) \widetilde{\psi}(x, \lambda)$ and $d \sigma(\lambda)=\left\{D_{s} h\left(l_{1}\right)\right\}^{-2} d \widetilde{\sigma}(\lambda)$, we obtain the conclusion from $(2.33)-(2.36)$.

\section{§3. Inverse local time for $\mathbb{D}_{s_{h}, m_{h}, 0}^{*}$}

Let $\mathbb{D}_{s, m, k}$ be the diffusion process on $I$ with generator $\mathcal{G}_{s, m, k}$ and with the end point $l_{i}$ being absorbing whenever it is $(s, m, k)$-regular $(i=1,2)$. We denote by $p(t, x, y)$ the transition probability density with respect to $d m$.

Let $h \in \mathcal{H}_{s, m, k, \beta}$ and let $\mathcal{G}_{s_{h}, m_{h}, 0}$ be the harmonic transform of $\mathcal{G}_{s, m, k}$ based on $h$. Let $\mathbb{D}_{s_{h}, m_{h}, 0}$ be the diffusion process on $I$ with generator $\mathcal{G}_{s_{h}, m_{h}, 0}$ and with the end point $l_{i}$ being absorbing whenever it is $\left(s_{h}, m_{h}, 0\right)$-regular $(i=1,2)$. We denote by $p^{(h)}(t, x, y)$ the transition probability density with respect to $d m_{h}$.

When $l_{i}$ is $\left(s_{h}, m_{h}, 0\right)$-regular, it is possible to pose the reflecting boundary condition at $l_{i}(i=1,2)$. Let $\mathbb{D}_{s_{h}, m_{h}, 0}^{*}=\left[X(t), P_{x}^{(h *)}\right]$ be the diffusion process on $I$ whose generator is $\mathcal{G}_{s_{h}, m_{h}, 0}^{*}$ with domain $D\left(\mathcal{G}_{s_{h}, m_{h}, 0}^{*}\right)$, that is, the end point $l_{i}$ is reflecting whenever it is $\left(s_{h}, m_{h}, 0\right)$-regular $(i=1,2)$. 
Now we assume that $l_{1}$ is $\left(s_{h}, m_{h}, 0\right)$-regular. We consider the local time $l^{(h)}(t, \xi)$, that is,

$$
\int_{0}^{t} f(X(u)) d u=\int_{I} l^{(h)}(t, \xi) d m_{h}(\xi), \quad t>0,
$$

for bounded continuous functions $f$ on $I$. We denote by $\tau^{(h *)}(t)$ the inverse local time $\left(l^{(h)}\right)^{-1}\left(t, l_{1}\right)$ at the end point $l_{1}$.

The following result is an immediate consequence of Proposition 2.3.

Proposition 3.1. Assume the following conditions hold:

$$
\begin{aligned}
& l_{1} \text { is }\left(s_{h}, m_{h}, 0\right) \text {-regular and reflecting. } \\
& s_{h}\left(l_{2}\right)=\infty, \text { or } l_{2} \text { is }\left(s_{h}, m_{h}, 0\right) \text {-regular and reflecting. }
\end{aligned}
$$

Then $\left[\tau^{(h *)}(t), t \geq 0\right]$ is a Lévy process and there is a Lévy measure density $n^{(h *)}(\xi)$ such that

$$
\begin{aligned}
E_{l_{1}}^{(h *)}\left[e^{-\lambda \tau^{(h *)}(t)}\right] & =\exp \left\{-t \int_{0}^{\infty}\left(1-e^{-\lambda \xi}\right) n^{(h *)}(\xi) d \xi\right\} \\
n^{(h *)}(\xi) & =\lim _{x \rightarrow l_{1}} q^{(h *)}(\xi, x) /\left\{s_{h}(x)-s_{h}\left(l_{1}\right)\right\}
\end{aligned}
$$

where $E_{l_{1}}^{(h *)}$ stands for the expectation with respect to $P_{l_{1}}^{(h *)}$, and

$$
\int_{0}^{t} q^{(h *)}(\xi, x) d \xi=P_{x}^{(h *)}\left(\sigma_{l_{1}}<t\right), \quad t>0, x \in I .
$$

In particular, if $s_{h}\left(l_{2}\right)=\infty$, then

$$
\begin{aligned}
n^{(h *)}(\xi) & =\int_{[0, \infty)} e^{-\xi \lambda} d \sigma^{(h)}(\lambda) \\
& =\lim _{x, y \rightarrow l_{1}} D_{s_{h}(x)} D_{s_{h}(y)} p^{(h)}(\xi, x, y), \quad \xi>0 .
\end{aligned}
$$

Note that $p^{(h)}(t, x, y)$ has the representation (2.30) and $d \sigma^{(h)}(\lambda)$ satisfies $(2.31)$.

Now we give a representation of $n^{(h *)}(\xi)$ in terms of data corresponding to the diffusion process $\mathbb{D}_{s, m, k}$. By Theorem 1.1 of [9], $l_{1}$ is $\left(s_{h}, m_{h}, 0\right)$-regular if and only if one of the following conditions is satisfied:

$$
\begin{aligned}
& l_{1} \text { is }(s, m, k) \text {-regular and } h\left(l_{1}\right) \in(0, \infty) . \\
& l_{1} \text { is an }(s, m, k) \text {-entrance point, } h\left(l_{1}\right)=\infty, \text { and }\left|m_{h}\left(l_{1}\right)\right|<\infty . \\
& l_{1} \text { is }(s, m, k) \text {-natural, } h\left(l_{1}\right)=\infty \text {, and }\left|m_{h}\left(l_{1}\right)\right|<\infty .
\end{aligned}
$$


In the case (3.6), we have the representation (2.2) with $d \sigma(\lambda)$ satisfying (2.3). In the case (3.7), we also have the representation (2.2) with $d \sigma(\lambda)$ satisfying $(2.3)$ and $\psi_{o}(x, \lambda)$ replaced by $\psi(x, \lambda)$, which is the solution of the integral equation $(2.5)$, by Proposition 2.1, Lemma 2.6, and Remark 2.7 .

Theorem 3.2. Let $h \in \mathcal{H}_{s, m, k, \beta}$. Assume one of (3.6)-(3.8) holds. Further assume that $l_{1}$ is reflecting and $s_{h}\left(l_{2}\right)=\infty$. Then $(3.3)$ holds true, and $n^{(h *)}(\xi)$ is given by (3.4) and (3.5). In particular, if (3.6) is satisfied, then

$$
\begin{aligned}
n^{(h *)}(\xi) & =h\left(l_{1}\right)^{2} e^{-\beta \xi} \int_{[0, \infty)} e^{-\xi \lambda} d \sigma(\lambda) \\
& =h\left(l_{1}\right)^{2} e^{-\beta \xi} \lim _{x, y \rightarrow l_{1}} D_{s(x)} D_{s(y)} p(\xi, x, y) .
\end{aligned}
$$

If (3.7) is satisfied, then

$$
\begin{aligned}
n^{(h *)}(\xi) & =D_{s} h\left(l_{1}\right)^{2} e^{-\beta \xi} \int_{[0, \infty)} e^{-\xi \lambda} d \sigma(\lambda) \\
& =D_{s} h\left(l_{1}\right)^{2} e^{-\beta \xi} \lim _{x, y \rightarrow l_{1}} p(\xi, x, y) .
\end{aligned}
$$

Proof. Since one of (3.6)-(3.8) holds, (3.1) is satisfied. Therefore by Proposition $3.1,(3.3)$ holds true, and $n^{(h *)}(\xi)$ is given by (3.4) and (3.5).

Assume (3.6) holds. Then we have (2.29), and by (5.14) of [10], $d \sigma^{(h)}(\lambda)$ is a Borel measure on $[\beta, \infty)$ and $d \sigma^{(h)}(\lambda)=h\left(l_{1}\right)^{2} d_{\lambda} \sigma(\lambda-\beta)$. Therefore (3.9) follows from (3.4). (3.10) follows from (2.2), (2.4), (2.8), and the dominated convergence theorem.

Assume (3.7) holds. Then we have (2.29), and by (5.18) of [10], $d \sigma^{(h)}(\lambda)$ is a Borel measure on $[\beta, \infty)$ and $d \sigma^{(h)}(\lambda)=\left\{D_{s} h\left(l_{1}\right)\right\}^{2} d_{\lambda} \sigma(\lambda-\beta)$. Therefore (3.11) follows from (3.4). (3.12) follows from (2.2), (2.5), (2.9), and the dominated convergence theorem.

Thus the proof is complete.

Finally we consider the conditions of Theorem 3.2. The following result shows that $h \in \mathcal{H}_{s, m, k, \beta}$ satisfying the conditions of Theorem 3.2 must be $g_{2}(x, \beta)$ whenever $l_{1}$ is $(s, m, k)$-regular or an $(s, m, k)$-entrance point.

Proposition 3.3. Suppose that $l_{1}$ is $(s, m, k)$-regular or an $(s, m, k)$-entrance point, and $k \neq 0$ or $\beta>0$. Let $h \in \mathcal{H}_{s, m, k, \beta}$. Then the following conditions are equivalent:

(i) (2.6) is satisfied and $h(x)=C g_{2}(x, \beta)$ for some positive constant $C$.

(ii) (3.6) or (3.7) is satisfied, and $s_{h}\left(l_{2}\right)=\infty$. 
Proof. Since the statement is obvious when $l_{1}$ is regular, we only prove it when $l_{1}$ is an entrance point.

(i) $\Rightarrow$ (ii). By Proposition 2.2, we get $s_{h}\left(l_{2}\right)=\infty$. By Lemma 2.6 and Remark 2.7, (2.6) is equivalent to $\int_{\left(l_{1}, c_{o}\right]} g_{2}(x, \beta)^{2} d m(x)<\infty$. Thus we obtain (3.7).

(ii) $\Rightarrow$ (i). By Lemma 2.6 and Remark 2.7, (3.7) implies (2.6).

We note that $h(x)=C_{1} g_{1}(x, \beta)+C_{2} g_{2}(x, \beta)$. If $C_{1}>0$, by using Lemma 2.2 we have

$$
\int_{\left(c_{o}, l_{2}\right)} h(x)^{-2} d s(x) \leq C_{1}^{-2} \int_{\left(c_{o}, l_{2}\right)} g_{1}(x, \beta)^{-2} d s(x)<\infty .
$$

This contradicts $s_{h}\left(l_{2}\right)=\infty$. Therefore $C_{1}=0$ and $C_{2}>0$.

Noting Remark 2.7, we easily obtain the following result, so we omit the proof.

Proposition 3.4. Assume that the killing measure is null, $l_{1}$ is $(s, m, 0)$-regular or an $(s, m, 0)$-entrance point, (2.6) is satisfied, and $s\left(l_{2}\right)<\infty$. Take $h(x)=$ $C\left\{s\left(l_{2}\right)-s(x)\right\}\left(\in \mathcal{H}_{s, m, 0,0}\right)$, where $C$ is a positive constant. Then (3.6) or (3.7) is satisfied, and $s_{h}\left(l_{2}\right)=\infty$.

\section{§4. Examples}

In this section, we present some interesting diffusion operators including the $\mathcal{L}_{i}$ 's given by (1.3)-(1.5) and (1.9). Then, using Proposition 2.3 and Theorem 3.2, we deduce the corresponding Lévy measure densities. Here we use the following functions.

The Bessel function $J_{\nu}(x)$ :

$$
J_{\nu}(x)=\left(\frac{x}{2}\right)^{\nu} \sum_{n=0}^{\infty} \frac{(-1)^{n}(x / 2)^{2 n}}{n ! \Gamma(\nu+n+1)} .
$$

The modified Bessel functions $I_{\nu}(x)$ and $K_{\nu}(x)$ :

$$
I_{\nu}(x)=\left(\frac{x}{2}\right)^{\nu} \sum_{n=0}^{\infty} \frac{(x / 2)^{2 n}}{n ! \Gamma(\nu+n+1)}, \quad \nu>-1 ;
$$

for $n$ an integer,

$$
\begin{aligned}
K_{n}(x)=K_{-n}(x)= & (-1)^{n+1} I_{n}(x)\{\gamma+\log (x / 2)\} \\
& +\frac{(-1)^{n}}{2} \sum_{k=0}^{\infty} \frac{(x / 2)^{n+2 k}}{k !(n+k) !}\left[\sum_{m=1}^{k} \frac{1}{m}+\sum_{m=1}^{k+n} \frac{1}{m}\right] \\
& +\frac{1}{2} \sum_{r=0}^{n-1}(-1)^{r} \frac{(n-r-1) !}{r !}\left(\frac{x}{2}\right)^{2 r-n},
\end{aligned}
$$


where $\gamma$ is Euler's constant, i.e. $\gamma=0.57721 \ldots$; in case $\nu$ is not an integer,

$$
K_{\nu}(x)=\frac{\pi}{2} \frac{I_{-\nu}(x)-I_{\nu}(x)}{\sin \nu \pi} .
$$

The Whittaker functions $M_{k, l}(x)$ and $W_{k, l}(x)$ :

$$
\begin{aligned}
M_{k, l}(x) & =x^{l+1 / 2} e^{-x / 2} M(l-k+1 / 2,2 l+1, x) \\
& =x^{l+1 / 2} e^{-x / 2} \sum_{n=0}^{\infty} \frac{\Gamma(2 l+1) \Gamma(l-k+n+1 / 2)}{\Gamma(2 l+n+1) \Gamma(l-k+1 / 2)} \frac{x^{n}}{n !}, \\
W_{k, l}(x) & =x^{l+1 / 2} e^{-x / 2} U(l-k+1 / 2,2 l+1, x) \\
& =\frac{\Gamma(-2 l)}{\Gamma(1 / 2-l-k)} M_{k, l}(x)+\frac{\Gamma(2 l)}{\Gamma(1 / 2+l-k)} M_{k,-l}(x),
\end{aligned}
$$

where

$$
\begin{aligned}
M(a, b, x) & =1+\sum_{k=1}^{\infty} \frac{a(a+1) \cdots(a+k-1) x^{k}}{b(b+1) \cdots(b+k-1) k !} \\
U(a, b, x) & =\frac{\pi}{\sin (\pi b)}\left[\frac{M(a, b, x)}{\Gamma(1+a-b) \Gamma(b)}-x^{1-b} \frac{M(1+a-b, 2-b, x)}{\Gamma(a) \Gamma(2-b)}\right] .
\end{aligned}
$$

Example 4.1 (Bessel process). Let us consider the following diffusion operator on $I=(0, \infty)$ :

$$
\mathcal{G}_{1}^{(\nu)}=\frac{1}{2} \frac{d^{2}}{d x^{2}}+\frac{2 \nu+1}{2 x} \frac{d}{d x},
$$

where $-\infty<\nu<\infty$. This is the Bessel operator, and the scale function $s_{1}^{(\nu)}$ and the speed measure $m_{1}^{(\nu)}$ are given by

$$
d s_{1}^{(\nu)}(x)=x^{-2 \nu-1} d x, \quad d m_{1}^{(\nu)}(x)=2 x^{2 \nu+1} d x .
$$

The killing measure is null. The end point 0 is:

- an $\left(s_{1}^{(\nu)}, m_{1}^{(\nu)}, 0\right)$-entrance point if $\nu \geq 0$,

- $\left(s_{1}^{(\nu)}, m_{1}^{(\nu)}, 0\right)$-regular if $-1<\nu<0$,

- an $\left(s_{1}^{(\nu)}, m_{1}^{(\nu)}, 0\right)$-exit point if $\nu \leq-1$.

Further

$$
\int_{0}^{1}\left\{s_{1}^{(\nu)}(1)-s_{1}^{(\nu)}(x)\right\}^{2} d m_{1}^{(\nu)}(x)<\infty \Leftrightarrow|\nu|<1 .
$$

The end point $\infty$ is $\left(s_{1}^{(\nu)}, m_{1}^{(\nu)}, 0\right)$-natural for all $\nu$, and in particular,

$$
s_{1}^{(\nu)}(\infty)=\infty \Leftrightarrow \nu \leq 0 .
$$


Let $\mathbb{D}_{1}^{(\nu)}$ be the diffusion process on $I$ with generator $\mathcal{G}_{1}^{(\nu)}$, and with the end point 0 being absorbing if $-1<\nu<0$. We denote by $p_{1}^{(\nu)}(t, x, y)$ the transition probability density with respect to $d m_{1}^{(\nu)}$. It is known (see [1, p. 134]) that

$$
p_{1}^{(\nu)}(t, x, y)=\frac{1}{2 t} \exp \left\{-\frac{x^{2}+y^{2}}{2 t}\right\}(x y)^{-\nu} I_{|\nu|}\left(\frac{x y}{t}\right),
$$

which is represented as the Laplace transform of Bessel functions,

$$
p_{1}^{(\nu)}(t, x, y)=\int_{0}^{\infty} e^{-\lambda t} \psi_{1}^{(\nu)}(x, \lambda) \psi_{1}^{(\nu)}(y, \lambda) \sigma_{1}^{(\nu)}(\lambda) d \lambda,
$$

where

$$
\begin{aligned}
\psi_{1}^{(\nu)}(x, \lambda) & = \begin{cases}\Gamma(\nu+1)(2 / \lambda)^{\nu / 2} x^{-\nu} J_{\nu}(\sqrt{2 \lambda} x), & \nu \geq 0, \\
\Gamma(|\nu|) 2^{-1}(2 / \lambda)^{|\nu| / 2} x^{|\nu|} J_{|\nu|}(\sqrt{2 \lambda} x), & \nu<0,\end{cases} \\
\sigma_{1}^{(\nu)}(\lambda) & = \begin{cases}2^{-\nu-1} \Gamma(\nu+1)^{-2} \lambda^{\nu}, & \nu \geq 0, \\
2^{1-|\nu|} \Gamma(|\nu|)^{-2} \lambda^{|\nu|}, & \nu<0,\end{cases}
\end{aligned}
$$

(see [6, p. 200]). For $\alpha>0$ we denote by $g_{1, i}^{(\nu)}(x, \alpha), i=1,2$, the functions satisfying (2.11)-(2.15) with $s=s_{1}^{(\nu)}, m=m_{1}^{(\nu)}$ and $k=0$. It is known (see [1, p. 133]) that

$$
g_{1,1}^{(\nu)}(x, \alpha)=x^{-\nu} I_{|\nu|}(\sqrt{2 \alpha} x), \quad g_{1,2}^{(\nu)}(x, \alpha)=x^{-\nu} K_{|\nu|}(\sqrt{2 \alpha} x) .
$$

Now we consider the Lévy measure densities corresponding to the inverse local time at the end point 0 .

(1) Let $-1<\nu<0$. Then the end point 0 is $\left(s_{1}^{(\nu)}, m_{1}^{(\nu)}, 0\right)$-regular. We pose the reflecting boundary condition at 0 . We denote by $\mathbb{D}_{1}^{(\nu, *)}$ the diffusion process with generator $\mathcal{G}_{1}^{(\nu)}$ and with the end point 0 being reflecting. We denote by $n_{1}^{(\nu, *)}$ the corresponding Lévy measure density. Since $s_{1}^{(\nu)}(\infty)=\infty$ by (4.4), from (2.24) we deduce

$$
\begin{aligned}
n_{1}^{(\nu, *)}(\xi) & =\lim _{x, y \rightarrow 0} D_{s_{1}^{(\nu)}(x)} D_{s_{1}^{(\nu)}(y)} p_{1}^{(\nu)}(\xi, x, y) \\
& =\int_{0}^{\infty} e^{-\xi \lambda} \sigma_{1}^{(\nu)}(\lambda) d \lambda=2^{-|\nu|+1} \frac{|\nu|}{\Gamma(|\nu|)} \xi^{-(|\nu|+1)} .
\end{aligned}
$$

(2) Let $-1<\nu<1$. Then the end point 0 is $\left(s_{1}^{(\nu)}, m_{1}^{(\nu)}, 0\right)$-regular or an $\left(s_{1}^{(\nu)}, m_{1}^{(\nu)}, 0\right)$-entrance point, and (2.6) is satisfied in view of (4.3). For $\beta>0$ we put $h_{1}^{(\nu, \beta)}(x)=(\beta / 2)^{|\nu| / 2} g_{1,2}^{(\nu)}(x, \beta)$ and denote by $\mathcal{G}_{1}^{(\nu, \beta)}$ the harmonic transform of $\mathcal{G}_{1}^{(\nu)}$ based on $h_{1}^{(\nu, \beta)} \in \mathcal{H}_{s_{1}^{(\nu)}, m_{1}^{(\nu)}, 0, \beta}$, that is,

$$
\mathcal{G}_{1}^{(\nu, \beta)}=\frac{1}{2} \frac{d^{2}}{d x^{2}}+\left\{\frac{1}{2 x}+\sqrt{2 \beta} \frac{K_{\nu}^{\prime}(\sqrt{2 \beta} x)}{K_{\nu}(\sqrt{2 \beta} x)}\right\} \frac{d}{d x},
$$


which is (1.3). Note that $\mathcal{G}_{1}^{(\nu, \beta)}=\mathcal{G}_{1}^{(-\nu, \beta)}$. The scale function $s_{1}^{(\nu, \beta)}$ and the speed measure $m_{1}^{(\nu, \beta)}$ are given by

$$
d s_{1}^{(\nu, \beta)}(x)=h_{1}^{(\nu, \beta)}(x)^{-2} d s_{1}^{(\nu)}(x), \quad d m_{1}^{(\nu, \beta)}(x)=h_{1}^{(\nu, \beta)}(x)^{2} d m_{2}^{(\nu)}(x) .
$$

By Proposition 3.3, (3.6) or (3.7) is satisfied, and $s_{1}^{(\nu, \beta)}(\infty)=\infty$. The end point 0 is $\left(s_{1}^{(\nu, \beta)}, m_{1}^{(\nu, \beta)}, 0\right)$-regular. We consider the diffusion process $\mathbb{D}_{1}^{(\nu, \beta, *)}$ with generator $\mathcal{G}_{1}^{(\nu, \beta)}$ and with the end point 0 being reflecting. Let $n_{1}^{(\nu, \beta, *)}$ be the corresponding Lévy measure density.

(2-i) Assume $\nu=0$. Since $D_{s_{1}^{(0)}} h_{1}^{(0, \beta)}(0)=-1$, (3.11) implies

$$
n_{1}^{(0, \beta, *)}(\xi)=e^{-\beta \xi} \int_{0}^{\infty} e^{-\xi \lambda} \sigma_{1}^{(0)}(\lambda) d \lambda=\frac{1}{2 \xi} e^{-\beta \xi} .
$$

(2-ii) Assume $-1<\nu<0$. Since $h_{1}^{(\nu, \beta)}(0)=\Gamma(|\nu|) / 2 \in(0, \infty)$, (3.9) leads to

$$
\begin{aligned}
n_{1}^{(\nu, \beta, *)}(\xi) & =h_{1}^{(\nu, \beta)}(0)^{2} e^{-\beta \xi} \int_{0}^{\infty} e^{-\xi \lambda} \sigma_{1}^{(\nu)}(\lambda) d \lambda \\
& =2^{-|\nu|-1} \Gamma(|\nu|+1) \xi^{-(|\nu|+1)} e^{-\beta \xi} .
\end{aligned}
$$

(2-iii) Assume $0<\nu<1$. Since $\mathcal{G}_{1}^{(\nu, \beta)}=\mathcal{G}_{1}^{(-\nu, \beta)}$, by using (4.7) we get

$$
n_{1}^{(\nu, \beta, *)}(\xi)=n_{1}^{(-\nu, \beta, *)}(\xi)=2^{-\nu-1} \Gamma(\nu+1) \xi^{-(\nu+1)} e^{-\beta \xi} .
$$

(4.6)-(4.8) show (1.6). As mentioned in Section 1, (1.6) was obtained by C. DonatiMartin and M. Yor [2] (see also [11]).

(3) Let $0<\nu<1$. Then $s_{1}^{(\nu)}(\infty)<\infty$ by (4.4). Therefore Proposition 3.4 leads to a special case corresponding to $\beta=0$.

We put $h_{1}^{(\nu, 0)}(x)=\left\{s_{1}^{(\nu)}(\infty)-s_{1}^{(\nu)}(x)\right\} /\left\{s_{1}^{(\nu)}(\infty)-s_{1}^{(\nu)}(1)\right\}=x^{-2 \nu}$. Denote by $\mathcal{G}_{1}^{(\nu, 0)}$ the harmonic transform of $\mathcal{G}_{1}^{(\nu)}$ based on $h_{1}^{(\nu, 0)} \in \mathcal{H}_{s_{1}^{(\nu)}, m_{1}^{(\nu)}, 0,0}$, that is,

$$
\mathcal{G}_{1}^{(\nu, 0)}=\frac{1}{2} \frac{d^{2}}{d x^{2}}+\frac{-2 \nu+1}{2 x} \frac{d}{d x} .
$$

We note that this coincides with $\mathcal{G}_{1}^{(-\nu)}$. The scale function $s_{1}^{(\nu, 0)}$ and the speed measure $m_{1}^{(\nu, 0)}$ are given by

$$
\begin{aligned}
d s_{1}^{(\nu, 0)}(x) & =h_{1}^{(\nu, 0)}(x)^{-2} d s_{1}^{(\nu)}(x)=x^{2 \nu-1} d x, \\
d m_{1}^{(\nu, 0)}(x) & =h_{1}^{(\nu, 0)}(x)^{2} d m_{1}^{(\nu)}(x)=2 x^{-2 \nu+1} d x .
\end{aligned}
$$

By Proposition 3.4, (3.7) is satisfied, and $s_{1}^{(\nu, 0)}(\infty)=\infty$. The end point 0 is $\left(s_{1}^{(\nu, 0)}, m_{1}^{(\nu, 0)}, 0\right)$-regular. We consider the diffusion process $\mathbb{D}_{1}^{(\nu, 0, *)}$ with generator 
$\mathcal{G}_{1}^{(\nu, 0)}$ and with the end point 0 being reflecting. Let $n_{1}^{(\nu, 0, *)}$ be the corresponding Lévy measure density. Since $\mathcal{G}_{1}^{(\nu, 0)}=\mathcal{G}_{1}^{(-\nu)}$, (4.5) yields

$$
n_{1}^{(\nu, 0, *)}(\xi)=2^{-|-\nu|+1}|-\nu| \Gamma(|-\nu|)^{-1} \xi^{-(|-\nu|+1)}=2^{-\nu+1} \frac{\nu}{\Gamma(\nu)} \xi^{-\nu-1},
$$

which coincides with $n_{1}^{(-\nu, *)}$.

Example 4.2 (Radial Ornstein-Uhlenbeck process). Let us consider the following diffusion operator on $I=(0, \infty)$ :

$$
\mathcal{G}_{2}^{(\nu, \kappa)}=\frac{1}{2} \frac{d^{2}}{d x^{2}}+\left(\frac{2 \nu+1}{2 x}-\kappa x\right) \frac{d}{d x},
$$

where $-\infty<\nu<\infty$ and $\kappa>0$. This is a radial Ornstein-Uhlenbeck operator, and the scale function $s_{2}^{(\nu, \kappa)}$ and the speed measure $m_{2}^{(\nu, \kappa)}$ are given by

$$
d s_{2}^{(\nu, \kappa)}(x)=x^{-2 \nu-1} e^{\kappa x^{2}} d x, \quad d m_{2}^{(\nu, \kappa)}(x)=2 x^{2 \nu+1} e^{-\kappa x^{2}} d x .
$$

The killing measure is null. The end point 0 is:

- an $\left(s_{2}^{(\nu, \kappa)}, m_{2}^{(\nu, \kappa)}, 0\right)$-entrance point if $\nu \geq 0$,

- $\left(s_{2}^{(\nu, \kappa)}, m_{2}^{(\nu, \kappa)}, 0\right)$-regular if $-1<\nu<0$,

- an $\left(s_{2}^{(\nu, \kappa)}, m_{2}^{(\nu, \kappa)}, 0\right)$-exit point if $\nu \leq-1$.

Further

$$
\int_{0}^{1}\left\{s_{2}^{(\nu, \kappa)}(1)-s_{2}^{(\nu, \kappa)}(x)\right\}^{2} d m_{2}^{(\nu, \kappa)}(x)<\infty \Leftrightarrow|\nu|<1 .
$$

The end point $\infty$ is always $\left(s_{2}^{(\nu, \kappa)}, m_{2}^{(\nu, \kappa)}, 0\right)$-natural for all $\nu$, and

$$
s_{2}^{(\nu, \kappa)}(\infty)=\infty \text {. }
$$

Let $\mathbb{D}_{2}^{(\nu, \kappa)}$ be the diffusion process on $I$ with generator $\mathcal{G}_{2}^{(\nu, \kappa)}$, and with the end point 0 being absorbing if $-1<\nu<0$. We denote by $p_{2}^{(\nu, \kappa)}(t, x, y)$ the transition probability density with respect to $d m_{2}^{(\nu, \kappa)}$. It is known (see [1, pp. 139-140]) that

$$
\begin{aligned}
& p_{2}^{(\nu, \kappa)}(t, x, y) \\
& \quad=\frac{\kappa}{2 x^{\nu} y^{\nu} \sinh (\kappa t)} \exp \left\{\kappa(\nu+1) t-\frac{\kappa e^{-\kappa t}\left(x^{2}+y^{2}\right)}{2 \sinh (\kappa t)}\right\} I_{|\nu|}\left(\frac{\kappa x y}{\sinh (\kappa t)}\right) .
\end{aligned}
$$

By (4.11), we see that

$$
\lim _{\kappa \rightarrow 0} p_{2}^{(\nu, \kappa)}(t, x, y)=p_{1}^{(\nu)}(t, x, y) .
$$


Since $\lim _{\kappa \rightarrow 0} s_{2}^{(\nu, \kappa)}(x)=s_{1}^{(\nu)}(x)$ and $\lim _{\kappa \rightarrow 0} m_{2}^{(\nu, \kappa)}(x)=m_{1}^{(\nu)}(x)$ for $x \in I,(4.12)$ also follows from Lemma 5.2 of [8]. Further we note the following: if $-1<\nu<0$,

$$
\begin{aligned}
\lim _{x, y \rightarrow 0} D_{s_{2}^{(\nu, \kappa)}(x)} D_{s_{2}^{(\nu, \kappa)}(y)} p_{2}^{(\nu, \kappa)}(\xi, x, y) & \\
= & 2^{-|\nu|+1} \frac{|\nu|}{\Gamma(|\nu|)}\left(\frac{\kappa}{\sinh (\kappa \xi)}\right)^{|\nu|+1} e^{\kappa(\nu+1) \xi}
\end{aligned}
$$

while if $\nu \geq 0$,

$$
\lim _{x, y \rightarrow 0} p_{2}^{(\nu, \kappa)}(\xi, x, y)=2^{-\nu-1} \frac{1}{\Gamma(\nu+1)}\left(\frac{\kappa}{\sinh (\kappa \xi)}\right)^{\nu+1} e^{\kappa(\nu+1) \xi} .
$$

For $\alpha>0$, we denote by $g_{2, i}^{(\nu, \kappa)}(x, \alpha), i=1,2$, the functions satisfying $(2.11)-(2.15)$ with $s=s_{2}^{(\nu, \kappa)}, m=m_{2}^{(\nu, \kappa)}$ and $k=0$. It is known (see [1, p. 139]) that

$$
\begin{aligned}
& g_{2,1}^{(\nu, \kappa)}(x, \alpha)=\frac{\alpha^{|\nu| / 2}}{2^{|\nu| / 2} \kappa^{(|\nu|+1) / 2} \Gamma(|\nu|+1)} x^{-\nu-1} e^{\kappa x^{2} / 2} M_{-\frac{\alpha}{2 \kappa}+\frac{\nu+1}{2}, \frac{|\nu|}{2}}\left(\kappa x^{2}\right), \\
& g_{2,2}^{(\nu, \kappa)}(x, \alpha)=\frac{\kappa^{|\nu| / 2-1 / 2}}{2^{1-|\nu| / 2} \alpha^{|\nu| / 2}} \Gamma\left(\frac{|\nu|}{2}-\frac{\nu}{2}+\frac{\alpha}{2 \kappa}\right) x^{-\nu-1} e^{\kappa x^{2} / 2} W_{-\frac{\alpha}{2 \kappa}+\frac{\nu+1}{2}, \frac{|\nu|}{2}}\left(\kappa x^{2}\right) .
\end{aligned}
$$

Now we consider the Lévy measure densities corresponding to the inverse local time at the end point 0 .

(1) Let $-1<\nu<0$. Then the end point 0 is $\left(s_{2}^{(\nu, \kappa)}, m_{2}^{(\nu, \kappa)}, 0\right)$-regular. We pose the reflecting boundary condition at 0 . We denote by $\mathbb{D}_{2}^{(\nu, \kappa, *)}$ the diffusion process with generator $\mathcal{G}_{2}^{(\nu, \kappa)}$ and with the end point 0 being reflecting. We denote by $n_{2}^{(\nu, \kappa, *)}$ the corresponding Lévy measure density. Since $s_{2}^{(\nu, \kappa)}(\infty)=\infty$ by $(4.10)$, from (2.24) and (4.13) we derive

$$
\begin{aligned}
n_{2}^{(\nu, \kappa, *)}(\xi) & =\lim _{x, y \rightarrow 0} D_{s_{2}^{(\nu, \kappa)}(x)} D_{s_{2}^{(\nu, \kappa)}(y)} p_{2}^{(\nu, \kappa)}(\xi, x, y) \\
& =2^{-|\nu|+1} \frac{|\nu|}{\Gamma(|\nu|)}\left(\frac{\kappa}{\sinh (\kappa \xi)}\right)^{|\nu|+1} e^{\kappa(\nu+1) \xi} .
\end{aligned}
$$

In view of Theorem 2.4 we get

$$
\lim _{\kappa \rightarrow 0} n_{2}^{(\nu, \kappa, *)}(\xi)=n_{1}^{(\nu, *)}(\xi) .
$$

We also note that this can be directly obtained from (4.5) and (4.15).

(2) Let $-1<\nu<1$. Then 0 is $\left(s_{2}^{(\nu, \kappa)}, m_{2}^{(\nu, \kappa)}, 0\right)$-regular or an $\left(s_{2}^{(\nu, \kappa)}, m_{2}^{(\nu, \kappa)}, 0\right)$ entrance point, and (2.6) is satisfied in view of (4.9). For $\beta>0$ we put $h_{2}^{(\nu, \kappa, \beta)}(x)=$ 
$(\beta / 2)^{|\nu| / 2} g_{2,2}^{(\nu, \kappa)}(x, \beta)$ and denote by $\mathcal{G}_{2}^{(\nu, \kappa, \beta)}$ the harmonic transform of $\mathcal{G}_{2}^{(\nu, \kappa)}$ based on $h_{2}^{(\nu, \kappa, \beta)} \in \mathcal{H}_{s_{2}^{(\nu, \kappa)}, m_{2}^{(\nu, \kappa)}, 0, \beta}$, that is,

$$
\mathcal{G}_{2}^{(\nu, \kappa, \beta)}=\frac{1}{2} \frac{d^{2}}{d x^{2}}+\left\{-\frac{1}{2 x}+2 \kappa x \frac{W_{-\frac{\beta}{2 \kappa}+\frac{\nu+1}{2}, \frac{|\nu|}{2}}^{\prime}\left(\kappa x^{2}\right)}{W_{-\frac{\beta}{2 \kappa}+\frac{\nu+1}{2}, \frac{|\nu|}{2}}\left(\kappa x^{2}\right)}\right\} \frac{d}{d x},
$$

which is (1.4). The scale function $s_{2}^{(\nu, \kappa, \beta)}$ and the speed measure $m_{2}^{(\nu, \kappa, \beta)}$ are given by

$$
d s_{2}^{(\nu, \kappa, \beta)}(x)=h_{2}^{(\nu, \kappa, \beta)}(x)^{-2} d s_{2}^{(\nu, \kappa)}(x), \quad d m_{2}^{(\nu, \kappa, \beta)}(x)=h_{2}^{(\nu, \kappa, \beta)}(x)^{2} d m_{2}^{(\nu, \kappa)}(x) .
$$

By Proposition 3.3, (3.6) or (3.7) is satisfied, and $s_{2}^{(\nu, \kappa, \beta)}(\infty)=\infty$. The end point 0 is $\left(s_{2}^{(\nu, \kappa, \beta)}, m_{2}^{(\nu, \kappa, \beta)}, 0\right)$-regular. We consider the diffusion process $\mathbb{D}_{2}^{(\nu, \kappa, \beta, *)}$ with generator $\mathcal{G}_{2}^{(\nu, \kappa, \beta)}$ and with the end point 0 being reflecting. Let $n_{2}^{(\nu, \kappa, \beta, *)}$ be the corresponding Lévy measure density.

(2-i) Assume $\nu=0$. Since $D_{s_{2}^{(0, \kappa)}} h_{2}^{(0, \kappa, \beta)}(0)=-1$ by $[7$, Problem 17, p. 279] and recursion (see [6, p. 73]), (3.12) and (4.14) imply

$$
\begin{aligned}
n_{2}^{(0, \kappa, \beta, *)}(\xi) & =D_{s_{2}^{(0, \kappa)}} h_{2}^{(0, \kappa, \beta)}(0)^{2} e^{-\beta \xi} \lim _{x, y \rightarrow 0} p_{2}^{(\nu, \kappa)}(\xi, x, y) \\
& =\frac{\kappa}{2 \sinh (\kappa \xi)} e^{(\kappa-\beta) \xi} .
\end{aligned}
$$

(2-ii) Assume $-1<\nu<0$. Since $h_{2}^{(\nu, \kappa, \beta)}(0)=\Gamma(|\nu|) / 2,(3.10)$ and (4.13) imply

$$
n_{2}^{(\nu, \kappa, \beta, *)}(\xi)=2^{-|\nu|-1} \Gamma(|\nu|+1)\left(\frac{\kappa}{\sinh (\kappa \xi)}\right)^{|\nu|+1} e^{\{\kappa(\nu+1)-\beta\} \xi} .
$$

(2-iii) Assume $0<\nu<1$. Since $D_{s_{2}^{(\nu, \kappa)}} h_{2}^{(\nu, \kappa, \beta)}(0)=-\Gamma(\nu+1)$ by recursion (see $[6$, p. 73]), (3.12) and (4.14) imply

$$
\begin{aligned}
n_{2}^{(\nu, \kappa, \beta, *)}(\xi) & =D_{s_{2}^{(0, \kappa)}} h_{2}^{(0, \kappa, \beta)}(0)^{2} e^{-\beta \xi} \lim _{x, y \rightarrow 0} p_{2}^{(\nu, \kappa)}(\xi, x, y) \\
& =2^{-\nu-1} \Gamma(\nu+1)\left(\frac{\kappa}{\sinh (\kappa \xi)}\right)^{\nu+1} e^{\{\kappa(\nu+1)-\beta\} \xi}
\end{aligned}
$$

(4.16)-(4.18) show (1.7). As mentioned in Section 1, (1.7) was obtained by C. Donati-Martin and M. Yor [3] in the case $-1<\nu<0$.

By using one of the limit theorems of $\left[1\right.$, p. 640], we see that $\lim _{\kappa \rightarrow 0} g_{2,2}^{(\nu, \kappa)}(x, \beta)$ $=g_{1,2}^{(\nu)}(x, \beta)$, and hence $\lim _{\kappa \rightarrow 0} h_{2}^{(\nu, \kappa, \beta)}(x)=h_{1}^{(\nu, \beta)}(x), \lim _{\kappa \rightarrow 0} s_{2}^{(\nu, \kappa, \beta)}(x)=s_{1}^{(\nu, \beta)}(x)$ and $\lim _{\kappa \rightarrow 0} m_{2}^{(\nu, \kappa, \beta)}(x)=m_{1}^{(\nu, \beta)}(x)$. Theorem 2.4 yields

$$
\lim _{\kappa \rightarrow 0} n_{2}^{(\nu, \kappa, \beta, *)}(\xi)=n_{1}^{(\nu, \beta, *)}(\xi) .
$$

(4.19) also follows from (4.6)-(4.8) and (4.16)-(4.18). 
We finally consider the special case $\beta=\kappa(\nu+1)>0$. Then $\mathcal{G}_{2}^{(\nu, \kappa, \beta)}$ reduces to

$$
\begin{aligned}
\mathcal{G}_{2}^{(\nu, \kappa, \kappa(\nu+1))} & =\frac{1}{2} \frac{d^{2}}{d x^{2}}+\left\{-\frac{1}{2 x}+2 \kappa x \frac{W_{0,|\nu| / 2}^{\prime}\left(\kappa x^{2}\right)}{W_{0,|\nu| / 2}\left(\kappa x^{2}\right)}\right\} \frac{d}{d x} \\
& =\frac{1}{2} \frac{d^{2}}{d x^{2}}+\left\{\frac{1}{2 x}+\kappa x \frac{K_{|\nu| / 2}^{\prime}\left(\kappa x^{2} / 2\right)}{K_{|\nu| / 2}\left(\kappa x^{2} / 2\right)}\right\} \frac{d}{d x},
\end{aligned}
$$

where we used [7, Problem 19 in p. 279]. By (4.16)-(4.18), the Lévy measure density corresponding to the inverse local time at 0 for $\mathbb{D}_{2}^{(\nu, \kappa, \kappa(\nu+1), *)}$ is

$$
n_{2}^{(\nu, \kappa, \kappa(\nu+1), *)}(\xi)=2^{-|\nu|-1} \Gamma(|\nu|+1)\left(\frac{\kappa}{\sinh (\kappa \xi)}\right)^{|\nu|+1}
$$

Example 4.3 (Radial Ornstein-Uhlenbeck process). Let us consider the following diffusion operator on $I=(0, \infty)$ :

$$
\mathcal{G}_{3}^{(\nu, \kappa)}=\frac{1}{2} \frac{d^{2}}{d x^{2}}+\left(\frac{2 \nu+1}{2 x}+\kappa x\right) \frac{d}{d x},
$$

where $-\infty<\nu<\infty$ and $\kappa>0$. This is also a radial Ornstein-Uhlenbeck operator, and the scale function $s_{3}^{(\nu, \kappa)}$ and the speed measure $m_{3}^{(\nu, \kappa)}$ are given by

$$
d s_{3}^{(\nu, \kappa)}(x)=x^{-2 \nu-1} e^{-\kappa x^{2}} d x, \quad d m_{3}^{(\nu, \kappa)}(x)=2 x^{2 \nu+1} e^{\kappa x^{2}} d x .
$$

The killing measure is null. The end point 0 is:

- an $\left(s_{3}^{(\nu, \kappa)}, m_{3}^{(\nu, \kappa)}, 0\right)$-entrance point if $\nu \geq 0$,

- $\left(s_{3}^{(\nu, \kappa)}, m_{3}^{(\nu, \kappa)}, 0\right)$-regular if $-1<\nu<0$,

- an $\left(s_{3}^{(\nu, \kappa)}, m_{3}^{(\nu, \kappa)}, 0\right)$-exit point if $\nu \leq-1$.

Further

$$
\int_{0}^{1}\left\{s_{3}^{(\nu, \kappa)}(1)-s_{3}^{(\nu, \kappa)}(x)\right\}^{2} d m_{3}^{(\nu, \kappa)}(x)<\infty \Leftrightarrow|\nu|<1 .
$$

The end point $\infty$ is always $\left(s_{3}^{(\nu, \kappa)}, m_{3}^{(\nu, \kappa)}, 0\right)$-natural for all $\nu$, and

$$
s_{3}^{(\nu, \kappa)}(\infty)<\infty
$$

Let $\mathbb{D}_{3}^{(\nu, \kappa)}$ be the diffusion process on $I$ with generator $\mathcal{G}_{3}^{(\nu, \kappa)}$, and with the end point 0 being absorbing if $-1<\nu<0$. We denote by $p_{3}^{(\nu, \kappa)}(t, x, y)$ the transition probability density with respect to $d m_{3}^{(\nu, \kappa)}$. It is known (see [1, pp. 139-140]) that

$$
\begin{aligned}
& p_{3}^{(\nu, \kappa)}(t, x, y) \\
& =\frac{\kappa}{2 x^{\nu} y^{\nu} \sinh (\kappa t)} \exp \left\{-\kappa(\nu+1) t-\frac{\kappa e^{\kappa t}\left(x^{2}+y^{2}\right)}{2 \sinh (\kappa t)}\right\} I_{|\nu|}\left(\frac{\kappa x y}{\sinh (\kappa t)}\right) .
\end{aligned}
$$


By (4.22),

$$
\lim _{\kappa \rightarrow 0} p_{3}^{(\nu, \kappa)}(t, x, y)=p_{1}^{(\nu)}(t, x, y) \text {. }
$$

Since $\lim _{\kappa \rightarrow 0} s_{3}^{(\nu, \kappa)}(x)=s_{1}^{(\nu)}(x)$ and $\lim _{\kappa \rightarrow 0} m_{3}^{(\nu, \kappa)}(x)=m_{1}^{(\nu)}(x)$ for $x \in I,(4.23)$ also follows from Lemma 5.2 of [8]. In the same way as for (4.13) and (4.14), we find that if $-1<\nu<0$,

$$
\begin{aligned}
\lim _{x, y \rightarrow 0} D_{s_{3}^{(\nu, \kappa)}(x)} D_{s_{3}^{(\nu, \kappa)}(y)} & p_{3}^{(\nu, \kappa)}(\xi, x, y) \\
& =2^{-|\nu|+1} \frac{|\nu|}{\Gamma(|\nu|)}\left(\frac{\kappa}{\sinh (\kappa \xi)}\right)^{|\nu|+1} e^{-\kappa(\nu+1) \xi},
\end{aligned}
$$

while if $\nu \geq 0$,

$$
\lim _{x, y \rightarrow 0} p_{3}^{(\nu, \kappa)}(\xi, x, y)=2^{-\nu-1} \frac{1}{\Gamma(\nu+1)}\left(\frac{\kappa}{\sinh (\kappa \xi)}\right)^{\nu+1} e^{-\kappa(\nu+1) \xi} .
$$

For $\alpha>0$ we denote by $g_{3, i}^{(\nu, \kappa)}(x, \alpha), i=1,2$, the functions satisfying $(2.11)-(2.15)$ with $s=s_{3}^{(\nu, \kappa)}, m=m_{3}^{(\nu, \kappa)}$ and $k=0$. It is known (see [1, p. 140] $]^{1}$ ) that

$$
\begin{aligned}
& g_{3,1}^{(\nu, \kappa)}(x, \alpha)=\frac{\alpha^{|\nu| / 2}}{2^{|\nu| / 2} \kappa^{(|\nu|+1) / 2} \Gamma(|\nu|+1)} x^{-\nu-1} e^{-\kappa x^{2} / 2} M_{-\frac{\alpha}{2 \kappa}-\frac{\nu+1}{2}, \frac{|\nu|}{2}}\left(\kappa x^{2}\right), \\
& g_{3,2}^{(\nu, \kappa)}(x, \alpha) \\
& \quad=\frac{\kappa^{|\nu| / 2-1 / 2}}{2^{1-|\nu| / 2} \alpha^{|\nu| / 2}} \Gamma\left(\frac{|\nu|}{2}+\frac{\nu}{2}+\frac{\alpha}{2 \kappa}+1\right) x^{-\nu-1} e^{-\kappa x^{2} / 2} W_{-\frac{\alpha}{2 \kappa}-\frac{\nu+1}{2}, \frac{|\nu|}{2}}\left(\kappa x^{2}\right) .
\end{aligned}
$$

Now we consider the Lévy measure densities corresponding to the inverse local time at the end point 0 . We assume $-1<\nu<1$. Then the end point 0 is $\left(s_{3}^{(\nu, \kappa)}, m_{3}^{(\nu, \kappa)}, 0\right)$-regular or an $\left(s_{3}^{(\nu, \kappa)}, m_{3}^{(\nu, \kappa)}, 0\right)$-entrance point, and (2.6) is satisfied by (4.20).

(1) For $\beta>0$ we put $h_{3}^{(\nu, \kappa, \beta)}(x)=(\beta / 2)^{|\nu| / 2} g_{3,2}^{(\nu, \kappa)}(x, \beta)$ and denote by $\mathcal{G}_{3}^{(\nu, \kappa, \beta)}$ the harmonic transform of $\mathcal{G}_{3}^{(\nu, \kappa)}$ based on $h_{3}^{(\nu, \kappa, \beta)} \in \mathcal{H}_{s_{3}^{(\nu, \kappa)}, m_{3}^{(\nu, \kappa)}, 0, \beta}$, that is,

$$
\mathcal{G}_{3}^{(\nu, \kappa, \beta)}=\frac{1}{2} \frac{d^{2}}{d x^{2}}+\left\{-\frac{1}{2 x}+2 \kappa x \frac{W_{-\frac{\beta}{2 \kappa}-\frac{\nu+1}{2}, \frac{|\nu|}{2}}^{\prime}\left(\kappa x^{2}\right)}{W_{-\frac{\beta}{2 \kappa}-\frac{\nu+1}{2}, \frac{|\nu|}{2}}\left(\kappa x^{2}\right)}\right\} \frac{d}{d x},
$$

which is (1.5). The scale function $s_{3}^{(\nu, \kappa, \beta)}$ and the speed measure $m_{3}^{(\nu, \kappa, \beta)}$ are given by

$$
d s_{3}^{(\nu, \kappa, \beta)}(x)=h_{3}^{(\nu, \kappa, \beta)}(x)^{-2} d s_{3}^{(\nu, \kappa)}(x), \quad d m_{3}^{(\nu, \kappa, \beta)}(x)=h_{3}^{(\nu, \kappa, \beta)}(x)^{2} d m_{3}^{(\nu, \kappa)}(x) .
$$

\footnotetext{
${ }^{1}$ Misprints in [1, p. 140]: minus signs are missing from exponents of Green functions in the case $\gamma<0$.
} 
By Proposition 3.3, (3.6) or (3.7) is satisfied, and $s_{3}^{(\nu, \kappa, \beta)}(\infty)=\infty$. The end point 0 is $\left(s_{3}^{(\nu, \kappa, \beta)}, m_{3}^{(\nu, \kappa, \beta)}, 0\right)$-regular. We consider the diffusion process $\mathbb{D}_{3}^{(\nu, \kappa, \beta, *)}$ with generator $\mathcal{G}_{3}^{(\nu, \kappa, \beta)}$ and with the end point 0 being reflecting. Let $n_{3}^{(\nu, \kappa, \beta, *)}$ be the corresponding Lévy measure density.

(1-i) Assume $\nu=0$. Since $D_{s_{3}^{(0, \kappa)}} h_{3}^{(0, \kappa, \beta)}(0)=-1$ by $[7$, Problem 17, p. 279] and recursion (see [6, p. 73]), (3.12) and (4.25) imply

$$
\begin{aligned}
n_{3}^{(0, \kappa, \beta, *)}(\xi) & =D_{s_{3}^{(0, \kappa)}} h_{3}^{(0, \kappa, \beta)}(0)^{2} e^{-\beta \xi} \lim _{x, y \rightarrow 0} p_{3}^{(\nu, \kappa)}(\xi, x, y) \\
& =\frac{\kappa}{2 \sinh (\kappa \xi)} e^{(-\kappa-\beta) \xi} .
\end{aligned}
$$

(1-ii) Assume $-1<\nu<0$. Since $h_{3}^{(\nu, \kappa, \beta)}(0)=\Gamma(|\nu|) / 2,(3.10)$ and (4.24) imply

$$
n_{3}^{(\nu, \kappa, \beta, *)}(\xi)=2^{-|\nu|-1} \Gamma(|\nu|+1)\left(\frac{\kappa}{\sinh (\kappa \xi)}\right)^{|\nu|+1} e^{\{-\kappa(\nu+1)-\beta\} \xi} .
$$

(1-iii) Assume $0<\nu<1$. Since $D_{s_{3}^{(\nu, \kappa)}} h_{3}^{(\nu, \kappa, \beta)}(0)=-\Gamma(\nu+1)$ by recursion (see $[6$, p. 73$]),(3.12)$ and (4.25) imply

$$
\begin{aligned}
n_{3}^{(\nu, \kappa, \beta, *)}(\xi) & =D_{s_{3}^{(0, \kappa)}} h_{3}^{(0, \kappa, \beta)}(0)^{2} e^{-\beta \xi} \lim _{x, y \rightarrow 0} p_{3}^{(\nu, \kappa)}(\xi, x, y) \\
& =2^{-\nu-1} \Gamma(\nu+1)\left(\frac{\kappa}{\sinh (\kappa \xi)}\right)^{\nu+1} e^{\{-\kappa(\nu+1)-\beta\} \xi} .
\end{aligned}
$$

By one of the limit theorems of [1, p. 640], we get $\lim _{\kappa \rightarrow 0} g_{3,2}^{(\nu, \kappa)}(x, \beta)=$ $g_{1,2}^{(\nu)}(x, \beta)$, and hence $\lim _{\kappa \rightarrow 0} s_{3}^{(\nu, \kappa, \beta)}(x)=s_{1}^{(\nu, \beta)}(x)$ and $\lim _{\kappa \rightarrow 0} m_{3}^{(\nu, \kappa, \beta)}(x)=$ $m_{1}^{(\nu, \beta)}(x)$. Theorem 2.4 yields

$$
\lim _{\kappa \rightarrow 0} n_{3}^{(\nu, \kappa, \beta, *)}(\xi)=n_{1}^{(\nu, \beta, *)}(\xi) .
$$

This also follows from (4.6)-(4.8) and (4.26)-(4.28).

(2) Since $s_{3}^{(\nu, \kappa)}(\infty)<\infty$ by (4.21), Proposition 3.4 leads to a special case corresponding to $\beta=0$. Put $h_{3}^{(\nu, \kappa, 0)}(x)=\left\{s_{3}^{(\nu, \kappa)}(\infty)-s_{3}^{(\nu, \kappa)}(x)\right\} /\left\{s_{3}^{(\nu, \kappa)}(\infty)-s_{3}^{(\nu, \kappa)}(1)\right\}$. Denote by $\mathcal{G}_{3}^{(\nu, \kappa, 0)}$ the harmonic transform of $\mathcal{G}_{3}^{(\nu, \kappa)}$ based on $h_{3}^{(\nu, \kappa, 0)} \in$ $\mathcal{H}_{s_{3}^{(\nu, \kappa)}, m_{3}^{(\nu, \kappa)}, 0,0}$, that is,

$$
\mathcal{G}_{3}^{(\nu, \kappa, 0)}=\frac{1}{2} \frac{d^{2}}{d x^{2}}+\left\{\frac{2 \nu+1}{2 x}+\kappa x-\frac{x^{-(2 \nu+1)} e^{-\kappa x^{2}}}{\int_{x}^{\infty} y^{-(2 \nu+1)} e^{-\kappa y^{2}} d y}\right\} \frac{d}{d x} .
$$

The scale function $s_{3}^{(\nu, \kappa, 0)}$ and the speed measure $m_{3}^{(\nu, \kappa, 0)}$ are given by

$$
d s_{3}^{(\nu, \kappa, 0)}(x)=h_{3}^{(\nu, \kappa, 0)}(x)^{-2} d s_{3}^{(\nu, \kappa)}(x), \quad d m_{3}^{(\nu, \kappa, 0)}(x)=h_{3}^{(\nu, \kappa, 0)}(x)^{2} d m_{3}^{(\nu, \kappa)}(x) .
$$


By Proposition 3.4, (3.6) or (3.7) is satisfied, and $s_{3}^{(\nu, \kappa, 0)}(\infty)=\infty$. The end point 0 is $\left(s_{3}^{(\nu, \kappa, 0)}, m_{3}^{(\nu, \kappa, 0)}, 0\right)$-regular. We consider the diffusion process $\mathbb{D}_{3}^{(\nu, \kappa, 0, *)}$ with generator $\mathcal{G}_{3}^{(\nu, \kappa, 0)}$ and with the end point 0 being reflecting. Let $n_{3}^{(\nu, \kappa, 0, *)}$ be the corresponding Lévy measure density.

(2-i) Assume $0 \leq \nu<1$. Since $D_{s_{3}^{(\nu, \kappa)}} h_{3}^{(\nu, \kappa, 0)}(0)=-\left\{s_{3}^{(\nu, \kappa)}(\infty)-s_{3}^{(\nu, \kappa)}(1)\right\}^{-1}$, (3.12) implies

$$
n_{3}^{(\nu, \kappa, 0, *)}(\xi)=\left\{s_{3}^{(\nu, \kappa)}(\infty)-s_{3}^{(\nu, \kappa)}(1)\right\}^{-2} 2^{-\nu-1} \frac{1}{\Gamma(\nu+1)}\left(\frac{\kappa}{\sinh (\kappa \xi)}\right)^{\nu+1} e^{-\kappa(\nu+1) \xi},
$$

where we used (4.25). We note that

$$
\lim _{\kappa \rightarrow 0} n_{3}^{(\nu, \kappa, 0, *)}(\xi)=2^{-\nu+1} \frac{\nu^{2}}{\Gamma(\nu+1)} \xi^{-\nu-1} .
$$

On the other hand,

$$
\lim _{\kappa \rightarrow 0} h_{3}^{(\nu, \kappa, 0)}(x)=x^{-2 \nu}
$$

and hence $\lim _{\kappa \rightarrow 0} s_{3}^{(\nu, \kappa, 0)}(x)=s_{1}^{(-\nu)}(x)$ and $\lim _{\kappa \rightarrow 0} m_{3}^{(\nu, \kappa, 0)}(x)=m_{1}^{(-\nu)}(x)$.

In the case $0<\nu<1$, the end point 0 is $\left(s_{1}^{(-\nu)}, m_{1}^{(-\nu)}, 0\right)$-regular, and hence by Theorem 2.4 ,

$$
\lim _{\kappa \rightarrow 0} n_{3}^{(\nu, \kappa, 0, *)}(\xi)=n_{1}^{(-\nu, *)}=2^{-\nu+1} \frac{\nu}{\Gamma(\nu)} \xi^{-\nu-1} .
$$

This shows (4.29) with $0<\nu<1$.

In the case $\nu=0$, the end point 0 is an $\left(s_{1}^{(0)}, m_{1}^{(0)}, 0\right)$-entrance point and hence the local time at 0 does not exist.

(2-ii) Assume $-1<\nu<0$. Since $h_{3}^{(\nu, \kappa, 0)}(0) \in(0, \infty)$, by (3.10) and $(4.24)$ we get

$$
n_{3}^{(\nu, \kappa, 0, *)}(\xi)=h_{3}^{(\nu, \kappa, 0)}(0)^{2} 2^{-|\nu|+1} \frac{|\nu|}{\Gamma(|\nu|)}\left(\frac{\kappa}{\sinh (\kappa \xi)}\right)^{|\nu|+1} e^{-\kappa(\nu+1) \xi}
$$

Since $\lim _{\kappa \rightarrow 0} h_{3}^{(\nu, \kappa, 0)}(0)=1$, we have $\lim _{\kappa \rightarrow 0} s_{3}^{(\nu, \kappa, 0)}(x)=s_{1}^{(\nu)}(x)$ and $\lim _{\kappa \rightarrow 0} m_{3}^{(\nu, \kappa, 0)}(x)=m_{1}^{(\nu)}(x)$. By Theorem 2.4 and (4.5),

$$
\lim _{\kappa \rightarrow 0} n_{3}^{(\nu, \kappa, 0, *)}(\xi)=n_{1}^{(\nu, *)}(\xi)=2^{-|\nu|+1} \frac{|\nu|}{\Gamma(|\nu|)} \xi^{-|\nu|-1} .
$$

This also follows from (4.30).

Example 4.4 (Squared Bessel process). Let us consider the following diffusion operator on $I=(0, \infty)$ :

$$
\mathcal{G}_{4}^{(\nu)}=2 x \frac{d^{2}}{d x^{2}}+(2 \nu+2) \frac{d}{d x}
$$


where $-\infty<\nu<\infty$. This is the squared Bessel operator, and the scale function $s_{4}^{(\nu)}$ and the speed measure $m_{4}^{(\nu)}$ are given by

$$
d s_{4}^{(\nu)}(x)=x^{-\nu-1} d x, \quad d m_{4}^{(\nu)}(x)=2^{-1} x^{\nu} d x .
$$

The killing measure is null. The end point 0 is:

- an $\left(s_{4}^{(\nu)}, m_{4}^{(\nu)}, 0\right)$-entrance point if $\nu \geq 0$,

- $\left(s_{4}^{(\nu)}, m_{4}^{(\nu)}, 0\right)$-regular if $-1<\nu<0$,

- an $\left(s_{4}^{(\nu)}, m_{4}^{(\nu)}, 0\right)$-exit point if $\nu \leq-1$.

Further

$$
\int_{0}^{1}\left\{s_{4}^{(\nu)}(1)-s_{4}^{(\nu)}(x)\right\}^{2} d m_{4}^{(\nu)}(x)<\infty \Leftrightarrow|\nu|<1 .
$$

The end point $\infty$ is $\left(s_{4}^{(\nu)}, m_{4}^{(\nu)}, 0\right)$-natural for all $\nu$, and

$$
s_{4}^{(\nu)}(\infty)=\infty \Leftrightarrow \nu \leq 0 .
$$

Let $\mathbb{D}_{4}^{(\nu)}$ be the diffusion process on $I$ with generator $\mathcal{G}_{4}^{(\nu)}$, and with the end point 0 being absorbing if $-1<\nu<0$. We denote by $p_{4}^{(\nu)}(t, x, y)$ the transition probability density with respect to $d m_{4}^{(\nu)}$. It is known (see [1, p. 136]) that

$$
p_{4}^{(\nu)}(t, x, y)=\frac{1}{t} \exp \left\{-\frac{x+y}{2 t}\right\}(x y)^{-\nu / 2} I_{|\nu|}\left(\frac{\sqrt{x y}}{t}\right) .
$$

In the same way as for the spectral representation of $p_{1}^{(\nu)}(t, x, y)$ in Example 4.1, we get

$$
p_{4}^{(\nu)}(t, x, y)=\int_{0}^{\infty} e^{-\lambda t} \psi_{4}^{(\nu)}(x, \lambda) \psi_{4}^{(\nu)}(y, \lambda) \sigma_{4}^{(\nu)}(\lambda) d \lambda,
$$

where

$$
\begin{aligned}
\psi_{4}^{(\nu)}(x, \lambda) & = \begin{cases}\Gamma(\nu+1)(2 / \lambda)^{\nu / 2} x^{-\nu / 2} J_{\nu}(\sqrt{2 \lambda x}), & \nu \geq 0, \\
\Gamma(|\nu|)(2 / \lambda)^{|\nu| / 2} x^{|\nu| / 2} J_{|\nu|}(\sqrt{2 \lambda x}), & \nu<0,\end{cases} \\
\sigma_{4}^{(\nu)}(\lambda) & = \begin{cases}2^{-\nu} \Gamma(\nu+1)^{-2} \lambda^{\nu}, & \nu \geq 0 \\
2^{-|\nu|} \Gamma(|\nu|)^{-2} \lambda^{|\nu|}, & \nu<0\end{cases}
\end{aligned}
$$

For $\alpha>0$ we denote by $g_{4, i}^{(\nu)}(x, \alpha), i=1,2$, the functions satisfying (2.11)-(2.15) with $s=s_{4}^{(\nu)}, m=m_{4}^{(\nu)}$ and $k=0$. It is known (see [1, p. 135]) that

$$
g_{4,1}^{(\nu)}(x, \alpha)=x^{-\nu / 2} I_{|\nu|}(\sqrt{2 \alpha x}), \quad g_{4,2}^{(\nu)}(x, \alpha)=x^{-\nu / 2} K_{|\nu|}(\sqrt{2 \alpha x}) .
$$

Now we consider the Lévy measure densities corresponding to the inverse local time at the end point 0 . 
(1) Let $-1<\nu<0$. Then the end point 0 is $\left(s_{4}^{(\nu)}, m_{4}^{(\nu)}, 0\right)$-regular. We pose the reflecting boundary condition at 0 . We denote by $\mathbb{D}_{4}^{(\nu, *)}$ the diffusion process with generator $\mathcal{G}_{4}^{(\nu)}$ and with the end point 0 being reflecting. We denote by $n_{4}^{(\nu, *)}$ the corresponding Lévy measure density. Since $s_{4}^{(\nu)}(\infty)=\infty$ by (4.32), from (2.24) we derive

$$
\begin{aligned}
n_{4}^{(\nu, *)}(\xi) & =\lim _{x, y \rightarrow 0} D_{s_{4}^{(\nu)}(x)} D_{s_{4}^{(\nu)}(y)} p_{4}^{(\nu)}(\xi, x, y) \\
& =\int_{0}^{\infty} e^{-\xi \lambda} \sigma_{4}^{(\nu)}(\lambda) d \lambda=2^{-|\nu|} \frac{|\nu|}{\Gamma(|\nu|)} \xi^{-(|\nu|+1)} .
\end{aligned}
$$

(2) Let $-1<\nu<1$. Then 0 is $\left(s_{4}^{(\nu)}, m_{4}^{(\nu)}, 0\right)$-regular or an $\left(s_{4}^{(\nu)}, m_{4}^{(\nu)}, 0\right)$ entrance point, and (2.6) is satisfied in view of (4.31). For $\beta>0$ we put $h_{4}^{(\nu, \beta)}(x)=(\beta / 2)^{|\nu| / 2} g_{4,2}^{(\nu)}(x, \beta)$ and denote by $\mathcal{G}_{4}^{(\nu, \beta)}$ the harmonic transform of $\mathcal{G}_{4}^{(\nu)}$ based on $h_{4}^{(\nu, \beta)} \in \mathcal{H}_{s_{4}^{(\nu)}, m_{4}^{(\nu)}, 0, \beta}$, that is,

$$
\mathcal{G}_{4}^{(\nu, \beta)}=2 x \frac{d^{2}}{d x^{2}}+2\left\{1+\sqrt{2 \beta x} \frac{K_{\nu}^{\prime}(\sqrt{2 \beta x})}{K_{\nu}(\sqrt{2 \beta x})}\right\} \frac{d}{d x} .
$$

The scale function $s_{4}^{(\nu, \beta)}$ and the speed measure $m_{4}^{(\nu, \beta)}$ are given by

$$
d s_{4}^{(\nu, \beta)}(x)=h_{4}^{(\nu, \beta)}(x)^{-2} d s_{4}^{(\nu)}(x), \quad d m_{4}^{(\nu, \beta)}(x)=h_{4}^{(\nu, \beta)}(x)^{2} d m_{4}^{(\nu)}(x) .
$$

By Proposition 3.3, (3.6) or (3.7) is satisfied, and $s_{4}^{(\nu, \beta)}(\infty)=\infty$. The end point 0 is $\left(s_{4}^{(\nu, \beta)}, m_{4}^{(\nu, \beta)}, 0\right)$-regular. We consider the diffusion process $\mathbb{D}_{4}^{(\nu, \beta, *)}$ with generator $\mathcal{G}_{4}^{(\nu, \beta)}$ and with the end point 0 being reflecting. Let $n_{4}^{(\nu, \beta, *)}$ be the corresponding Lévy measure density.

(2-i) Assume $\nu=0$. Since $D_{s_{4}^{(0)}} h_{4}^{(0, \beta)}(0)=-1 / 2,(3.11)$ implies

$$
n_{4}^{(0, \beta, *)}(\xi)=\frac{1}{4} e^{-\beta \xi} \int_{[0, \infty)} e^{-\xi \lambda} \sigma_{4}^{(0)}(\lambda) d \lambda=\frac{1}{4 \xi} e^{-\beta \xi}
$$

(2-ii) Assume $-1<\nu<0$. Since $h_{4}^{(\nu, \beta)}(0)=\Gamma(-\nu) / 2$, (3.9) leads to

$$
\begin{aligned}
n_{4}^{(\nu, \beta, *)}(\xi) & =h_{4}^{(\nu, \beta)}(0)^{2} e^{-\beta \xi} \int_{0}^{\infty} e^{-\xi \lambda} \sigma_{4}^{(\nu)}(\lambda) d \lambda \\
& =2^{\nu-2} \Gamma(|\nu|+1) \xi^{\nu-1} e^{-\beta \xi}
\end{aligned}
$$

(2-iii) Assume $0<\nu<1$. Since $\mathcal{G}_{4}^{(\nu, \beta)}=\mathcal{G}_{4}^{(-\nu, \beta)}$, by using (4.35) we get

$$
n_{4}^{(\nu, \beta, *)}(\xi)=n_{4}^{(-\nu, \beta, *)}(\xi)=2^{-\nu-2} \Gamma(\nu+1) \xi^{-\nu-1} e^{-\beta \xi} .
$$

(3) Let $0<\nu<1$. Then $s_{4}^{(\nu)}(\infty)<\infty$ by (4.32). Therefore Proposition 3.4 leads to a special case corresponding to $\beta=0$. We put $h_{4}^{(\nu, 0)}(x)=\left\{s_{4}^{(\nu)}(\infty)-\right.$ 
$\left.s_{4}^{(\nu)}(x)\right\} /\left\{s_{4}^{(\nu)}(\infty)-s_{4}^{(\nu)}(1)\right\}=x^{-\nu}$. Denote by $\mathcal{G}_{4}^{(\nu, 0)}$ the harmonic transform of $\mathcal{G}_{4}^{(\nu)}$ based on $h_{4}^{(\nu, 0)} \in \mathcal{H}_{s_{4}^{(\nu)}, m_{4}^{(\nu)}, 0,0}$, that is,

$$
\mathcal{G}_{4}^{(\nu, 0)}=2 x \frac{d^{2}}{d x^{2}}+(-2 \nu+2) \frac{d}{d x} .
$$

We note that this coincides with $\mathcal{G}_{4}^{(-\nu)}$. The scale function $s_{4}^{(\nu, 0)}$ and the speed measure $m_{4}^{(\nu, 0)}$ are given by

$$
\begin{aligned}
d s_{4}^{(\nu, 0)}(x) & =h_{4}^{(\nu, 0)}(x)^{-2} d s_{4}^{(\nu)}(x)=x^{\nu-1} d x=d s_{4}^{(-\nu)}(x), \\
d m_{4}^{(\nu, 0)}(x) & =h_{4}^{(\nu, 0)}(x)^{2} d m_{4}^{(\nu)}(x)=2^{-1} x^{-\nu} d x=d m_{4}^{(-\nu)}(x) .
\end{aligned}
$$

By Proposition 3.4, (3.7) is satisfied, and $s_{4}^{(\nu, 0)}(\infty)=\infty$. The end point 0 is $\left(s_{4}^{(\nu, 0)}, m_{4}^{(\nu, 0)}, 0\right)$-regular. We consider the diffusion process $\mathbb{D}_{4}^{(\nu, 0, *)}$ with generator $\mathcal{G}_{4}^{(\nu, 0)}$ and with the end point 0 being reflecting. Let $n_{4}^{(\nu, 0, *)}$ be the corresponding Lévy measure density. Since $\mathcal{G}_{4}^{(\nu, 0)}=\mathcal{G}_{4}^{(-\nu)},(4.33)$ yields

$$
n_{4}^{(\nu, 0, *)}(\xi)=2^{-\nu} \frac{\nu}{\Gamma(\nu)} \xi^{-(\nu+1)}=n_{4}^{(-\nu, *)}(\xi) .
$$

Example 4.5 (Squared radial Ornstein-Uhlenbeck process). Let us consider the following diffusion operator on $I=(0, \infty)$ :

$$
\mathcal{G}_{5}^{(\nu, \kappa)}=2 x \frac{d^{2}}{d x^{2}}+(2 \nu+2-2 \kappa x) \frac{d}{d x},
$$

where $-\infty<\nu<\infty$ and $\kappa>0$. This is a squared radial Ornstein-Uhlenbeck operator, and the scale function $s_{5}^{(\nu, \kappa)}$ and the speed measure $m_{5}^{(\nu, \kappa)}$ are

$$
d s_{5}^{(\nu, \kappa)}(x)=x^{-\nu-1} e^{\kappa x} d x, \quad d m_{5}^{(\nu, \kappa)}(x)=2^{-1} x^{\nu} e^{-\kappa x} d x .
$$

The killing measure is null. The end point 0 is:

- an $\left(s_{5}^{(\nu, \kappa)}, m_{5}^{(\nu, \kappa)}, 0\right)$-entrance point if $\nu \geq 0$,

- $\left(s_{5}^{(\nu, \kappa)}, m_{5}^{(\nu, \kappa)}, 0\right)$-regular if $-1<\nu<0$,

- an $\left(s_{5}^{(\nu, \kappa)}, m_{5}^{(\nu, \kappa)}, 0\right)$-exit point if $\nu \leq-1$.

Further

$$
\int_{0}^{1}\left\{s_{5}^{(\nu, \kappa)}(1)-s_{5}^{(\nu, \kappa)}(x)\right\}^{2} d m_{5}^{(\nu, \kappa)}(x)<\infty \Leftrightarrow|\nu|<1 .
$$

The end point $\infty$ is $\left(s_{5}^{(\nu, \kappa)}, m_{5}^{(\nu, \kappa)}, 0\right)$-natural for all $\nu$ and

$$
s_{5}^{(\nu, \kappa)}(\infty)=\infty
$$


Let $\mathbb{D}_{5}^{(\nu, \kappa)}$ be the diffusion process on $I$ with generator $\mathcal{G}_{5}^{(\nu, \kappa)}$, and with the end point 0 being absorbing if $-1<\nu<0$. We denote by $p_{5}^{(\nu, \kappa)}(t, x, y)$ the transition probability density with respect to $d m_{5}^{(\nu, \kappa)}$. It is known (see [1, p. 142]) that

$$
\begin{aligned}
& p_{5}^{(\nu, \kappa)}(t, x, y) \\
& \quad=\frac{\kappa}{\sinh (\kappa t)}(x y)^{-\nu / 2} \exp \left\{\kappa(\nu+1) t-\frac{\kappa e^{-\kappa t}(x+y)}{2 \sinh (\kappa t)}\right\} I_{|\nu|}\left(\frac{\kappa \sqrt{x y}}{\sinh (\kappa t)}\right) .
\end{aligned}
$$

By (4.39),

$$
\lim _{\kappa \rightarrow 0} p_{5}^{(\nu, \kappa)}(t, x, y)=p_{4}^{(\nu)}(t, x, y)
$$

Since $\lim _{\kappa \rightarrow 0} s_{5}^{(\nu, \kappa)}(x)=s_{4}^{(\nu)}(x)$ and $\lim _{\kappa \rightarrow 0} m_{5}^{(\nu, \kappa)}(x)=m_{4}^{(\nu)}(x)$ for $x \in I,(4.40)$ also follows from Lemma 5.2 of [8]. Further we note that if $-1<\nu<0$,

$$
\begin{aligned}
\lim _{x, y \rightarrow 0} D_{s_{5}^{(\nu, \kappa)}(x)} D_{s_{5}^{(\nu, \kappa)}(y)} p_{5}^{(\nu, \kappa)} & (\xi, x, y) \\
& =2^{-|\nu|} \frac{|\nu|}{\Gamma(|\nu|)}\left(\frac{\kappa}{\sinh (\kappa \xi)}\right)^{|\nu|+1} e^{\kappa(\nu+1) \xi},
\end{aligned}
$$

while if $\nu \geq 0$

$$
\lim _{x, y \rightarrow 0} p_{6}^{(\nu, \kappa)}(\xi, x, y)=2^{-\nu} \frac{1}{\Gamma(\nu+1)}\left(\frac{\kappa}{\sinh (\kappa \xi)}\right)^{\nu+1} e^{\kappa(\nu+1) \xi} .
$$

For $\alpha>0$ we denote by $g_{5, i}^{(\nu, \kappa)}(x, \alpha), i=1,2$, the functions satisfying (2.11)-(2.15) with $s=s_{5}^{\nu, \kappa}, m=m_{5}^{\nu, \kappa}$ and $k=0$. It is known (see [1, pp. 141-142]) that

$$
\begin{aligned}
& g_{5,1}^{(\nu, \kappa)}(x, \alpha)=\frac{\alpha^{|\nu| / 2}}{2^{|\nu| / 2} \Gamma(|\nu|+1) \kappa^{(|\nu|+1) / 2}} x^{-\frac{\nu+1}{2}} e^{\kappa x / 2} M_{-\frac{\alpha}{2 \kappa}+\frac{\nu+1}{2}, \frac{|\nu|}{2}}(\kappa x), \\
& g_{5,2}^{(\nu, \kappa)}(x, \alpha)=\frac{2^{|\nu| / 2-1} \kappa^{(|\nu|-1) / 2}}{\alpha^{|\nu| / 2}} \Gamma\left(\frac{\alpha}{2 \kappa}+\frac{|\nu|}{2}-\frac{\nu}{2}\right) x^{-\frac{\nu+1}{2}} e^{\kappa x / 2} W_{-\frac{\alpha}{2 \kappa}+\frac{\nu+1}{2}, \frac{|\nu|}{2}}(\kappa x) .
\end{aligned}
$$

Now we consider the Lévy measure densities corresponding to the inverse local time at the end point 0 .

(1) Let $-1<\nu<0$. Then the end point 0 is $\left(s_{5}^{(\nu, \kappa)}, m_{5}^{(\nu, \kappa)}, 0\right)$-regular. We pose the reflecting boundary condition at 0 . We denote by $\mathbb{D}_{5}^{(\nu, \kappa, *)}$ the diffusion process with generator $\mathcal{G}_{5}^{(\nu, \kappa)}$ and with the end point 0 being reflecting. We denote by $n_{5}^{(\nu, \kappa, *)}$ the corresponding Lévy measure density. Since $s_{5}^{(\nu, \kappa)}(\infty)=\infty$ by (4.38), from (2.24) and (4.41) we derive

$$
\begin{aligned}
n_{5}^{(\nu, \kappa, *)}(\xi) & =\lim _{x, y \rightarrow 0} D_{s_{5}^{(\nu, \kappa)}(x)} D_{s_{5}^{(\nu, \kappa)}(y)} p_{5}^{(\nu, \kappa)}(\xi, x, y) \\
& =\frac{2^{-|\nu|}|\nu|}{\Gamma(|\nu|)}\left(\frac{\kappa}{\sinh (\kappa \xi)}\right)^{|\nu|+1} e^{\kappa(\nu+1) \xi} .
\end{aligned}
$$


(2) Let $-1<\nu<1$. Then 0 is $\left(s_{5}^{(\nu, \kappa)}, m_{5}^{(\nu, \kappa)}, 0\right)$-regular or an $\left(s_{5}^{(\nu, \kappa)}, m_{5}^{(\nu, \kappa)}, 0\right)$ entrance point, and (2.6) is satisfied in view of (4.37). For $\beta>0$ we put $h_{5}^{(\nu, \kappa, \beta)}(x)=(\beta / 2)^{|\nu| / 2} g_{5,2}^{(\nu, \kappa)}(x, \beta)$ and denote by $\mathcal{G}_{5}^{(\nu, \kappa, \beta)}$ the harmonic transform of $\mathcal{G}_{5}^{(\nu, \kappa)}$ based on $h_{5}^{(\nu, \kappa, \beta)} \in \mathcal{H}_{s_{5}^{(\nu, \kappa)}, m_{5}^{(\nu, \kappa)}, 0, \beta}$, that is,

$$
\mathcal{G}_{5}^{(\nu, \kappa, \beta)}=2 x \frac{d^{2}}{d x^{2}}+4 \kappa x \frac{W_{-\frac{\beta}{2 \kappa}+\frac{\nu+1}{2}, \frac{|\nu|}{2}}^{\prime}(\kappa x)}{W_{-\frac{\beta}{2 \kappa}+\frac{\nu+1}{2}, \frac{|\nu|}{2}}(\kappa x)} \frac{d}{d x} \text {. }
$$

The scale function $s_{5}^{(\nu, \kappa, \beta)}$ and the speed measure $m_{5}^{(\nu, \kappa, \beta)}$ are given by

$$
d s_{5}^{(\nu, \kappa, \beta)}(x)=h_{5}^{(\nu, \kappa, \beta)}(x)^{-2} d s_{5}^{(\nu, \kappa)}(x), \quad d m_{5}^{(\nu, \kappa, \beta)}(x)=h_{5}^{(\nu, \kappa, \beta)}(x)^{2} d m_{5}^{(\nu, \kappa)}(x) .
$$

By Proposition 3.3, (3.6) or (3.7) is satisfied, and $s_{5}^{(\nu, \kappa, \beta)}(\infty)=\infty$. Therefore the end point 0 is $\left(s_{5}^{(\nu, \kappa, \beta)}, m_{5}^{(\nu, \kappa, \beta)}, 0\right)$-regular. We consider the diffusion process $\mathbb{D}_{5}^{(\nu, \kappa, \beta, *)}$ with generator $\mathcal{G}_{5}^{(\nu, \kappa, \beta)}$ and with the end point 0 being reflecting. Let $n_{5}^{(\nu, \kappa, \beta, *)}$ be the corresponding Lévy measure density.

(2-i) Assume $\nu=0$. Since $D_{s_{5}^{(0, \kappa)}} h_{5}^{(0, \kappa, \beta)}(0)=-1 / 2$ by [7, Problem 17, p. 279] and recursion (see [6, p. 73]), (3.12) and (4.42) imply

$$
n_{5}^{(0, \kappa, \beta, *)}(\xi)=\frac{\kappa}{4 \sinh (\kappa \xi)} e^{(\kappa-\beta) \xi}
$$

(2-ii) Assume $-1<\nu<0$. Since $h_{5}^{(\nu, \kappa, \beta)}(0)=\Gamma(|\nu|) / 2$, (3.10) and (4.41) lead to

$$
n_{5}^{(\nu, \kappa, \beta, *)}(\xi)=2^{-|\nu|-2} \Gamma(|\nu|+1)\left(\frac{\kappa}{\sinh (\kappa \xi)}\right)^{|\nu|+1} e^{(\kappa(\nu+1)-\beta) \xi}
$$

(2-iii) Assume $0<\nu<1$. Since $D_{s_{5}^{(\nu, \kappa)}} h_{5}^{(\nu, \kappa, \beta)}(0)=-\Gamma(1+\nu) / 2$ by recursion (see $[6$, p. 73]), (3.12) and (4.42) imply

$$
n_{5}^{(\nu, \kappa, \beta, *)}(\xi)=2^{-\nu-2} \Gamma(\nu+1)\left(\frac{\kappa}{\sinh (\kappa \xi)}\right)^{\nu+1} e^{(\kappa(\nu+1)-\beta) \xi} .
$$

By using one of the limit theorems of $[1$, p. 640], we see that $\lim _{\kappa \rightarrow 0} g_{5,2}^{(\nu, \kappa)}(x, \beta)=g_{4,2}^{(\nu)}(x, \beta)$, and hence $\lim _{\kappa \rightarrow 0} h_{5}^{(\nu, \kappa, \beta)}(x)=h_{4}^{(\nu, \beta)}(x)$, $\lim _{\kappa \rightarrow 0} s_{5}^{(\nu, \kappa, \beta)}(x)=s_{4}^{(\nu, \beta)}(x)$ and $\lim _{\kappa \rightarrow 0} m_{5}^{(\nu, \kappa, \beta)}(x)=m_{4}^{(\nu, \beta)}(x)$. Theorem 2.4 yields

$$
\lim _{\kappa \rightarrow 0} n_{5}^{(\nu, \kappa, \beta, *)}(\xi)=n_{4}^{(\nu, \beta, *)}(\xi) .
$$

(4.46) also follows from (4.34)-(4.36) and (4.43)-(4.45). 
We finally consider the special case $\beta=\kappa(\nu+1)>0$. Then $\mathcal{G}_{5}^{(\nu, \kappa, \beta)}$ reduces to

$$
\begin{aligned}
\mathcal{G}_{5}^{(\nu, \kappa, \kappa(\nu+1))} & =2 x \frac{d^{2}}{d x^{2}}+4 \kappa x \frac{W_{0,|\nu| / 2}^{\prime}(\kappa x)}{W_{0,|\nu| / 2}(\kappa x)} \frac{d}{d x} \\
& =2 x \frac{d^{2}}{d x^{2}}+\left\{1+\kappa x \frac{K_{|\nu| / 2}^{\prime}(\kappa x / 2)}{K_{|\nu| / 2}(\kappa x / 2)}\right\} \frac{d}{d x},
\end{aligned}
$$

where we used [7, Problem 19 in p. 279]. By (4.43), (4.44), and (4.45), the Lévy measure density corresponding to the inverse local time at 0 for $\mathbb{D}_{5}^{(\nu, \kappa, \kappa(\nu+1), *)}$ is

$$
n_{5}^{(\nu, \kappa, \kappa(\nu+1), *)}(\xi)=2^{-|\nu|-2} \Gamma(|\nu|+1)\left(\frac{\kappa}{\sinh (\kappa \xi)}\right)^{|\nu|+1} .
$$

Example 4.6 (Squared radial Ornstein-Uhlenbeck process). Let us consider the following diffusion operator on $I=(0, \infty)$ :

$$
\mathcal{G}_{6}^{(\nu, \kappa)}=2 x \frac{d^{2}}{d x^{2}}+(2 \nu+2+2 \kappa x) \frac{d}{d x},
$$

where $-\infty<\nu<\infty$ and $\kappa>0$. This is also a squared radial Ornstein-Uhlenbeck operator, and the scale function $s_{6}^{(\nu, \kappa)}$ and the speed measure $m_{6}^{(\nu, \kappa)}$ are given by

$$
d s_{6}^{(\nu, \kappa)}(x)=x^{-\nu-1} e^{-\kappa x} d x, \quad d m_{6}^{(\nu, \kappa)}(x)=2^{-1} x^{\nu} e^{\kappa x} d x .
$$

The killing measure is null. The end point 0 is:

- an $\left(s_{6}^{(\nu, \kappa)}, m_{6}^{(\nu, \kappa)}, 0\right)$-entrance point if $\nu \geq 0$,

- $\left(s_{6}^{(\nu, \kappa)}, m_{6}^{(\nu, \kappa)}, 0\right)$-regular if $-1<\nu<0$,

- an $\left(s_{6}^{(\nu, \kappa)}, m_{6}^{(\nu, \kappa)}, 0\right)$-exit point if $\nu \leq-1$.

Further

$$
\int_{0}^{1}\left\{s_{6}^{(\nu, \kappa)}(1)-s_{6}^{(\nu, \kappa)}(x)\right\}^{2} d m_{6}^{(\nu, \kappa)}(x)<\infty \Leftrightarrow|\nu|<1 .
$$

The end point $\infty$ is $\left(s_{6}^{(\nu, \kappa)}, m_{6}^{(\nu, \kappa)}, 0\right)$-natural for all $\nu$ and

$$
s_{6}^{(\nu, \kappa)}(\infty)<\infty .
$$

Let $\mathbb{D}_{6}^{(\nu, \kappa)}$ be the diffusion process on $I$ with generator $\mathcal{G}_{6}^{(\nu, \kappa)}$, and with the end point 0 being absorbing if $-1<\nu<0$. We denote by $p_{6}^{(\nu, \kappa)}(t, x, y)$ the transition probability density with respect to $d m_{6}^{(\nu, \kappa)}$. It is known (see [1, p. 142]) that

$$
\begin{aligned}
& p_{6}^{(\nu, \kappa)}(t, x, y) \\
= & \frac{\kappa}{\sinh (\kappa t)}(x y)^{-\nu / 2} \exp \left\{-\kappa(\nu+1) t-\frac{\kappa e^{\kappa t}(x+y)}{2 \sinh (\kappa t)}\right\} I_{|\nu|}\left(\frac{\kappa \sqrt{x y}}{\sinh (\kappa t)}\right) .
\end{aligned}
$$


By (4.50),

$$
\lim _{\kappa \rightarrow 0} p_{6}^{(\nu, \kappa)}(t, x, y)=p_{4}^{(\nu)}(t, x, y) .
$$

Since $\lim _{\kappa \rightarrow 0} s_{6}^{(\nu, \kappa)}(x)=s_{4}^{(\nu)}(x)$ and $\lim _{\kappa \rightarrow 0} m_{6}^{(\nu, \kappa)}(x)=m_{4}^{(\nu)}(x)$ for $x \in I,(4.51)$ also follows from Lemma 5.2 of [8].

In the same way as for (4.41) and (4.42), we find that if $-1<\nu<0$,

$$
\begin{aligned}
\lim _{x, y \rightarrow 0} D_{s_{6}^{(\nu, \kappa)}(x)} D_{s_{6}^{(\nu, \kappa)}(y)} p_{6}^{(\nu, \kappa)}(\xi, x, y) \\
=2^{-|\nu|} \frac{|\nu|}{\Gamma(|\nu|)}\left(\frac{\kappa}{\sinh (\kappa \xi)}\right)^{|\nu|+1} e^{-\kappa(\nu+1) \xi},
\end{aligned}
$$

while if $\nu \geq 0$,

$$
\lim _{x, y \rightarrow 0} p_{6}^{(\nu, \kappa)}(\xi, x, y)=2^{-\nu} \frac{1}{\Gamma(\nu+1)}\left(\frac{\kappa}{\sinh (\kappa \xi)}\right)^{\nu+1} e^{-\kappa(\nu+1) \xi} .
$$

For $\alpha>0$ we denote by $g_{6, i}^{(\nu, \kappa)}(x, \alpha), i=1,2$, the functions satisfying (2.11)-(2.15) with $s=s_{6}^{(\nu, \kappa)}, m=m_{6}^{(\nu, \kappa)}$ and $k=0$. It is known (see [1, p. 142] $]^{2}$ ) that

$$
\begin{aligned}
& g_{6,1}^{(\nu, \kappa)}(x, \alpha)=\frac{\alpha^{|\nu| / 2}}{2^{|\nu| / 2} \kappa^{(|\nu|+1) / 2} \Gamma(|\nu|+1)} x^{-\frac{\nu+1}{2}} e^{-\kappa x / 2} M_{-\frac{\alpha}{2 \kappa}-\frac{\nu+1}{2}, \frac{|\nu|}{2}}(\kappa x), \\
& g_{6,2}^{(\nu, \kappa)}(x, \alpha)=\frac{\kappa^{\frac{|\nu|-1}{2}}}{2^{1-\frac{|\nu|}{2}} \alpha^{\frac{|\nu|}{2}}} \Gamma\left(\frac{|\nu|}{2}+\frac{\nu}{2}+\frac{\alpha}{2 \kappa}+1\right) x^{-\frac{\nu+1}{2}} e^{-\kappa x / 2} W_{-\frac{\alpha}{2 \kappa}-\frac{\nu+1}{2}, \frac{|\nu|}{2}}(\kappa x) .
\end{aligned}
$$

Now we consider the Lévy measure densities corresponding to the inverse local time at the end point 0 .

In the following we assume $-1<\nu<1$. Then the end point 0 is $\left(s_{6}^{(\nu, \kappa)}, m_{6}^{(\nu, \kappa)}, 0\right)$-regular or an $\left(s_{6}^{(\nu, \kappa)}, m_{6}^{(\nu, \kappa)}, 0\right)$-entrance point, and $(2.6)$ is satisfied in view of (4.48).

(1) For $\beta>0$, we put $h_{6}^{(\nu, \kappa, \beta)}(x)=(\beta / 2)^{|\nu| / 2} g_{6,2}^{(\nu, \kappa)}(x, \beta)$ and denote by $\mathcal{G}_{6}^{(\nu, \kappa, \beta)}$ the harmonic transform of $\mathcal{G}_{6}^{(\nu, \kappa)}$ based on $h_{6}^{(\nu, \kappa, \beta)} \in \mathcal{H}_{s_{6}^{(\nu, \kappa)}, m_{6}^{(\nu, \kappa)}, 0, \beta}$, that is,

$$
\mathcal{G}_{6}^{(\nu, \kappa, \beta)}=2 x \frac{d^{2}}{d x^{2}}+4 \kappa x \frac{W_{-\frac{\beta}{2 \kappa}-\frac{\nu+1}{2}, \frac{|\nu|}{2}}^{\prime}(\kappa x)}{W_{-\frac{\beta}{2 \kappa}-\frac{\nu+1}{2}, \frac{|\nu|}{2}}(\kappa x)} \frac{d}{d x} .
$$

The scale function $s_{6}^{(\nu, \kappa, \beta)}$ and the speed measure $m_{6}^{(\nu, \kappa, \beta)}$ are given by

$$
d s_{6}^{(\nu, \kappa, \beta)}(x)=h_{6}^{(\nu, \kappa, \beta)}(x)^{-2} d s_{6}^{(\nu, \kappa)}(x), \quad d m_{6}^{(\nu, \kappa, \beta)}(x)=h_{6}^{(\nu, \kappa, \beta)}(x)^{2} d m_{6}^{(\nu, \kappa)}(x) .
$$

${ }^{2}$ Misprints in [1, p. 142]: there are unnecessary minus signs in exponents of Green functions in the case $\gamma<0$. 
By Proposition 3.3, (3.6) or (3.7) is satisfied, and $s_{6}^{(\nu, \kappa, \beta)}(\infty)=\infty$. The end point 0 is $\left(s_{6}^{(\nu, \kappa, \beta)}, m_{6}^{(\nu, \kappa, \beta)}, 0\right)$-regular. We consider the diffusion process $\mathbb{D}_{6}^{(\nu, \kappa, \beta, *)}$ with generator $\mathcal{G}_{6}^{(\nu, \kappa, \beta)}$ and with the end point 0 being reflecting. Let $n_{6}^{(\nu, \kappa, \beta, *)}$ be the corresponding Lévy measure density.

(1-i) Assume $\nu=0$. Since $D_{s_{6}^{(0, \kappa)}} h_{6}^{(0, \kappa, \beta)}(0)=-1 / 2$ by $[7$, Problem 17, p. 279] and recursion (see [6, p. 73]), (3.12) and (4.53) imply

$$
n_{6}^{(0, \kappa, \beta, *)}(\xi)=\frac{\kappa}{4 \sinh (\kappa \xi)} e^{(-\kappa-\beta) \xi} .
$$

(1-ii) Assume $-1<\nu<0$. Since $h_{6}^{(\nu, \kappa, \beta)}(0)=\Gamma(|\nu|) / 2,(3.10)$ and (4.52) lead to

$$
n_{6}^{(\nu, \kappa, \beta, *)}(\xi)=2^{-|\nu|-2} \Gamma(|\nu|+1)\left(\frac{\kappa}{\sinh (\kappa \xi)}\right)^{|\nu|+1} e^{(-\kappa(\nu+1)-\beta) \xi} .
$$

(1-iii) Assume $0<\nu<1$. Since $D_{s_{6}^{(\nu, \kappa)}} h_{6}^{(\nu, \kappa, \beta)}(0)=-\Gamma(\nu+1) / 2$ by recursion (see $[6$, p. 73$]),(3.12)$ and (4.53) imply

$$
n_{6}^{(\nu, \kappa, \beta, *)}(\xi)=2^{-\nu-2} \Gamma(\nu+1)\left(\frac{\kappa}{\sinh (\kappa \xi)}\right)^{\nu+1} e^{(-\kappa(\nu+1)-\beta) \xi} .
$$

By one of the limit theorems of $\left[1\right.$, p. 640], we get $\lim _{\kappa \rightarrow 0} g_{6,2}^{(\nu, \kappa)}(x, \beta)=g_{4,2}^{(\nu)}(x, \beta)$, and hence $\lim _{\kappa \rightarrow 0} s_{6}^{(\nu, \kappa, \beta)}(x)=s_{4}^{(\nu, \beta)}(x)$ and $\lim _{\kappa \rightarrow 0} m_{6}^{(\nu, \kappa, \beta)}(x)=m_{4}^{(\nu, \beta)}(x)$. By Theorem 2.4,

$$
\lim _{\kappa \rightarrow 0} n_{6}^{(\nu, \kappa, \beta, *)}(\xi)=n_{4}^{(\nu, \beta, *)}(\xi) .
$$

This also follows from (4.34)-(4.36) and (4.54)-(4.56).

(2) Since $s_{6}^{(\nu, \kappa)}(\infty)<\infty$ by (4.49), Proposition 3.4 leads to a special case corresponding to $\beta=0$. Put $h_{6}^{(\nu, \kappa, 0)}(x)=\left\{s_{6}^{(\nu, \kappa)}(\infty)-s_{6}^{(\nu, \kappa)}(x)\right\} /\left\{s_{6}^{(\nu, \kappa)}(\infty)-\right.$ $\left.s_{6}^{(\nu, \kappa)}(1)\right\}$. Denote by $\mathcal{G}_{6}^{(\nu, \kappa, 0)}$ the harmonic transform of $\mathcal{G}_{6}^{(\nu, \kappa)}$ based on $h_{6}^{(\nu, \kappa, 0)} \in$ $\mathcal{H}_{s_{6}^{(\nu, \kappa)}, m_{6}^{(\nu, \kappa)}, 0,0}$, that is,

$$
\mathcal{G}_{6}^{(\nu, \kappa, 0)}=2 x \frac{d^{2}}{d x^{2}}+\left\{2 \nu+2+2 \kappa x-\frac{4 x^{-\nu} e^{-\kappa x}}{\int_{x}^{\infty} y^{-(\nu+1)} e^{-\kappa y} d y}\right\} \frac{d}{d x} .
$$

The scale function $s_{6}^{(\nu, \kappa, 0)}$ and the speed measure $m_{6}^{(\nu, \kappa, 0)}$ are given by

$$
d s_{6}^{(\nu, \kappa, 0)}(x)=h_{6}^{(\nu, \kappa, 0)}(x)^{-2} d s_{6}^{(\nu, \kappa)}(x), \quad d m_{6}^{(\nu, \kappa, 0)}(x)=h_{6}^{(\nu, \kappa, 0)}(x)^{2} d m_{6}^{(\nu, \kappa)}(x) .
$$

By Proposition 3.4, (3.6) or (3.7) is satisfied, and $s_{6}^{(\nu, \kappa, 0)}(\infty)=\infty$. The end point 0 is $\left(s_{6}^{(\nu, \kappa, 0)}, m_{6}^{(\nu, \kappa, 0)}, 0\right)$-regular. We consider the diffusion process $\mathbb{D}_{6}^{(\nu, \kappa, 0, *)}$ with generator $\mathcal{G}_{6}^{(\nu, \kappa, 0)}$ and with the end point 0 being reflecting. Let $n_{6}^{(\nu, \kappa, 0, *)}$ be the corresponding Lévy measure density. 
(2-i) Assume $0 \leq \nu<1$. Since $D_{s_{6}^{(\nu, \kappa)}} h_{6}^{(\nu, \kappa, 0)}(0)=-\left\{s_{6}^{(\nu, \kappa)}(\infty)-s_{6}^{(\nu, \kappa)}(1)\right\}^{-1}$, (3.12) implies

$$
n_{6}^{(\nu, \kappa, 0, *)}(\xi)=\left\{s_{6}^{(\nu, \kappa)}(\infty)-s_{6}^{(\nu, \kappa)}(1)\right\}^{-2} 2^{-\nu} \frac{1}{\Gamma(\nu+1)}\left(\frac{\kappa}{\sinh (\kappa \xi)}\right)^{\nu+1} e^{-\kappa(\nu+1) \xi},
$$

where we used (4.52). We note that

$$
\lim _{\kappa \rightarrow 0} n_{6}^{(\nu, \kappa, 0, *)}(\xi)=2^{-\nu} \frac{\nu^{2}}{\Gamma(\nu+1)} \xi^{-\nu-1} .
$$

On the other hand,

$$
\lim _{\kappa \rightarrow 0} h_{6}^{(\nu, \kappa, 0)}(x)=x^{-\nu},
$$

and hence $\lim _{\kappa \rightarrow 0} s_{6}^{(\nu, \kappa, 0)}=s_{4}^{(-\nu)}$ and $\lim _{\kappa \rightarrow 0} m_{6}^{(\nu, \kappa, 0)}=m_{4}^{(-\nu)}$.

In the case $0<\nu<1$, the end point 0 is $\left(s_{4}^{(-\nu)}, m_{4}^{(-\nu)}, 0\right)$-regular, and hence

$$
\lim _{\kappa \rightarrow 0} n_{6}^{(\nu, \kappa, 0, *)}(\xi)=n_{4}^{(-\nu, *)}(\xi)=2^{-\nu} \frac{\nu^{2}}{\Gamma(\nu+1)} \xi^{-\nu-1} .
$$

This shows (4.57) with $0<\nu<1$.

In the case $\nu=0$, the end point 0 is an $\left(s_{4}^{(0)}, m_{4}^{(0)}, 0\right)$-entrance point and hence the local time at the end point 0 does not exist.

(2-ii) Assume $-1<\nu<0$. Since $h_{6}^{(\nu, \kappa, 0)}(0) \in(0, \infty)$, by (3.10) and (4.52) we get

$$
n_{6}^{(\nu, \kappa, 0, *)}(\xi)=h_{6}^{(\nu, \kappa, 0)}(0)^{2} 2^{-|\nu|} \frac{|\nu|}{\Gamma(|\nu|)}\left(\frac{\kappa}{\sinh (\kappa \xi)}\right)^{|\nu|+1} e^{-\kappa(\nu+1) \xi} .
$$

As $\lim _{\kappa \rightarrow 0} h_{6}^{(\nu, \kappa, 0)}(x)=1$, we have $\lim _{\kappa \rightarrow 0} s_{6}^{(\nu, \kappa, 0)}(x)=s_{4}^{(\nu)}$ and $\lim _{\kappa \rightarrow 0} m_{6}^{(\nu, \kappa, 0)}(x)$ $=m_{4}^{(\nu)}$. By Theorem 2.4 and (4.33),

$$
\lim _{\kappa \rightarrow 0} n_{6}^{(\nu, \kappa, 0, *)}(\xi)=n_{4}^{(\nu, *)}(\xi)=2^{-|\nu|} \frac{|\nu|}{\Gamma(|\nu|)} \xi^{-|\nu|-1} .
$$

This also follows from (4.58).

Example 4.7 (Brownian motion). Let us consider the following diffusion operator on $I=(0, a)$, where $0<a<\infty$ :

$$
\mathcal{G}_{7}^{(a)}=\frac{1}{2} \frac{d^{2}}{d x^{2}} .
$$

This is the generator of Brownian motion on $(0, a)$, and the scale function $s_{7}^{(a)}$ and the speed measure $m_{7}^{(a)}$ are given by

$$
d s_{7}^{(a)}(x)=d x, \quad d m_{7}^{(a)}(x)=2 d x .
$$

The killing measure is null. The end points 0 and $a$ are both $\left(s_{7}^{(a)}, m_{7}^{(a)}, 0\right)$-regular. 
Let $\mathbb{D}_{7}^{(a)}$ be the Brownian motion on $I$ with generator $\mathcal{G}_{7}^{(a)}$, and with the end points 0 and $a$ being both absorbing. We denote by $p_{7}^{(a)}(t, x, y)$ the transition probability density with respect to $d m_{7}^{(a)}$. It is known (see [1, p. 122]) that

$$
\begin{aligned}
p_{7}^{(a)}(t, x, y) & =\frac{1}{a} \sum_{n=1}^{\infty} e^{-n^{2} \pi^{2} t /\left(2 a^{2}\right)} \sin (n \pi x / a) \sin (n \pi y / a) \\
& =\int_{(0, \infty)} e^{-\lambda t} \psi_{7}^{(a)}(x, \lambda) \psi_{7}^{(a)}(y, \lambda) d \sigma_{7}^{(a)}(\lambda),
\end{aligned}
$$

where

$$
\psi_{7}^{(a)}\left(x, \lambda_{n}\right)=\frac{a}{n \pi} \sin (n \pi x / a), \quad d \sigma_{7}^{(a)}(\lambda)=\sum_{n=1}^{\infty} \frac{n^{2} \pi^{2}}{a^{3}} \delta_{\lambda_{n}}(d \lambda),
$$

$\lambda_{n}=n^{2} \pi^{2} /\left(2 a^{2}\right)$, and $\delta_{p}(d \lambda)$ is the unit measure concentrated at $p$. For $\alpha>0$, we denote by $g_{7, i}^{(a)}(x, \alpha), i=1,2$, the function satisfying $(2.11)-(2.15)$ with $s=s_{7}^{(a)}$, $m=m_{7}^{(a)}$ and $k=0$. It is easy to see that

$$
g_{7,1}^{(a)}(x, \alpha)=e^{\sqrt{2 \alpha} x}-e^{-\sqrt{2 \alpha} x}, \quad g_{7,2}^{(a)}(x, \alpha)=e^{\sqrt{2 \alpha}(a-x)}-e^{-\sqrt{2 \alpha}(a-x)} .
$$

Now we consider the Lévy measure densities corresponding to the inverse local time at the end point 0 . For $\beta>0$ we put $h_{7}^{(a)}(x)=C g_{7,2}^{(a)}(x, \beta)$, where $C=g_{7,2}^{(a)}(0, \beta)^{-1}$ and denote by $\mathcal{G}_{7}^{(a, \beta)}$ the harmonic transform of $\mathcal{G}_{7}^{(a)}$ based on $h_{7}^{(a, \beta)} \in \mathcal{H}_{s_{7}^{(a)}, m_{7}^{(a)}, 0, \beta}$, that is,

$$
\mathcal{G}_{7}^{(a, \beta)}=\frac{1}{2} \frac{d^{2}}{d x^{2}}-\sqrt{2 \beta} \frac{e^{2 \sqrt{2 \beta}(a-x)}+1}{e^{2 \sqrt{2 \beta}(a-x)}-1} \frac{d}{d x},
$$

which is (1.9). The scale function $s_{7}^{(a, \beta)}$ and the speed measure $m_{7}^{(a, \beta)}$ are given by

$$
d s_{7}^{(a, \beta)}(x)=h_{7}^{(a, \beta)}(x)^{-2} d s_{7}^{(a)}(x), \quad d m_{7}^{(a, \beta)}(x)=h_{7}^{(a, \beta)}(x)^{2} d m_{7}^{(a)}(x) .
$$

Since $h_{7}^{(a)}(0)=1$, by Proposition 3.3, (3.6) is satisfied, and $s_{7}^{(a, \beta)}(a)=\infty$. The end point 0 is $\left(s_{7}^{(a, \beta)}, m_{7}^{(a, \beta)}, 0\right)$-regular. We consider the diffusion process $\mathbb{D}_{7}^{(a, \beta, *)}$ with generator $\mathcal{G}_{7}^{(a, \beta)}$ and with the end point 0 being reflecting. Let $n_{7}^{(a, \beta, *)}$ be the corresponding Lévy measure density. By (3.9),

$$
n_{7}^{(a, \beta, *)}(\xi)=e^{-\beta \xi} \int_{(0, \infty)} e^{-\xi \lambda} d \sigma_{7}^{(a)}(\lambda)=e^{-\beta \xi} \sum_{n=1}^{\infty} e^{-\xi \lambda_{n}} \frac{n^{2} \pi^{2}}{a^{3}}
$$

This shows (1.10). 
We note that

$$
\lim _{a \rightarrow \infty} n_{7}^{(a, \beta, *)}(\xi)=\sqrt{\pi / 2} e^{-\beta \xi} \xi^{-3 / 2}
$$

Indeed, for $\Lambda>0$,

$$
\begin{aligned}
\int_{(0, \Lambda]} d \sigma_{7}^{(a)}(\lambda)=\frac{\pi^{2}}{a^{3}} \sum_{n: \lambda_{n} \leq \Lambda} n^{2}=\frac{\pi^{2}}{a^{3}} \sum_{n=1}^{\Lambda_{0}} n^{2} & =\frac{\pi^{2}}{a^{3}}\left(\frac{1}{3} \Lambda_{0}^{3}+\frac{1}{2} \Lambda_{0}^{2}+\frac{1}{6} \Lambda_{0}\right) \\
& \rightarrow \frac{2 \sqrt{2}}{3 \pi} \Lambda^{3 / 2} \quad \text { as } a \rightarrow \infty
\end{aligned}
$$

where $\Lambda_{0}$ indicates the integral part of $\sqrt{2 \Lambda} a / \pi$. Thus we have (4.59). We can also show (4.59) by using Theorem 2.4. Here is a proof. Let us consider the diffusion operator

$$
\mathcal{G}_{7}^{(\uparrow, \beta)}=\frac{1}{2} \frac{d^{2}}{d x^{2}}-\sqrt{2 \beta} \frac{d}{d x} .
$$

The scale function $s_{7}^{(\uparrow, \beta)}$ and the speed measure $m_{7}^{(\uparrow, \beta)}$ are given by

$$
d s_{7}^{(\uparrow, \beta)}(x)=e^{2 \sqrt{2 \beta} x} d x, \quad d m_{7}^{(\uparrow, \beta)}(x)=2 e^{-2 \sqrt{2 \beta} x} d x .
$$

The killing measure is null. The end point 0 is $\left(s_{7}^{(\uparrow, \beta)}, m_{7}^{(\uparrow, \beta)}, 0\right)$-regular. Let $\mathbb{D}_{7}^{(\uparrow, \beta, *)}$ be the diffusion process on $(0, \infty)$ with generator $\mathcal{G}_{7}^{(\uparrow, \beta)}$, and with the end point 0 being reflecting. We denote by $n_{7}^{(\uparrow, \beta, *)}(\xi)$ the corresponding Lévy measure density. By using [10, Example 6.1] and (2.24), we have

$$
n_{7}^{(\uparrow, \beta, *)}(\xi)=\int_{\beta}^{\infty} e^{-\lambda \xi} \sigma_{7}^{(\uparrow, \beta)}(\lambda) d \lambda=\sqrt{\pi / 2} e^{-\beta \xi} \xi^{-3 / 2} .
$$

As $\lim _{a \rightarrow \infty} h_{7}^{(a, \beta)}(x)=e^{-\sqrt{2 \beta} x}, \lim _{a \rightarrow \infty} s_{7}^{(a, \beta)}(x)=s_{7}^{(\uparrow, \beta)}(x)$, and $\lim _{a \rightarrow \infty} m_{7}^{(a, \beta)}(x)$ $=m_{7}^{(\uparrow, \beta)}(x)$, by Theorem 2.4 we obtain

$$
\lim _{a \rightarrow \infty} n_{7}^{(a, \beta, *)}(\xi)=n_{7}^{(\uparrow, \beta, *)}(\xi) .
$$

This shows (4.59).

\section{Acknowledgements}

The second author was partially supported by Grant-in-Aid for Scientific Research

(C) 22540132 .

\section{References}

[1] A. N. Borodin and P. Salminen, Handbook of Brownian motion-facts and formulae, Birkhäuser, Basel, 2002. Zbl 1012.60003 MR 1912205 
[2] C. Donati-Martin and M. Yor, Some explicit Krein representations of certain subordinators, including the Gamma processes, Publ. RIMS Kyoto Univ. 42 (2006), 879-895. Zbl 1123.60028 MR 2289152

[3] C. Donati-Martin and M. Yor, Further examples of explicit Krein representations of certain subordinators. Publ. RIMS Kyoto Univ. 43 (2007), 315-328. Zbl 1129.60042 MR 2341013

[4] W. Feller, The parabolic differential equations and the associated semi-groups of transformations, Ann. of Math. 55 (1952), 468-519. Zbl 0047.09303 MR 0047886

[5] K. Itô and H. P. McKean, Jr., Diffusion processes and their sample paths, Springer, New York, 1974. Zbl 0285.60063 MR 0345224

[6] S. Moriguchi, K. Udagawa and S. Hitotsumatsu, Mathematical formulas. III, Iwanami, 1960 (in Japanese).

[7] N. N. Lebedev, Spectral functions and their applications, Dover Publ., New York, 1972.

[8] Y. Ogura, One-dimensional bi-generalized diffusion processes, J. Math. Soc. Japan 41 (1989), 213-242. Zbl 0701.60078 MR 0984748

[9] T. Takemura, State of boundaries for harmonic transforms of one-dimensional generalized diffusion processes, Annual Reports Grad. School Humanities Sci. Nara Women's Univ. 25 (2019), 285-294.

[10] T. Takemura and M. Tomisaki, $h$ transform of one-dimensional generalized diffusion operators, Kyushu J. Math. 66 (2012), 171-191. Zbl pre06135998 MR 2962397

[11] M. Tomisaki, Intrinsic ultracontractivity and small perturbation for one-dimensional generalized diffusion operators, J. Funct. Anal. 251 (2007), 289-324. Zbl 1135.47044 MR 2353708

[12] M. Tomisaki, Inverse local times of harmonic transformed Bessel processes, Annual Reports Grad. School Humanities Sci. Nara Women's Univ. 22 (2011), 269-279. 